\title{
STAT6/Arg1 promotes microglia/ macrophage efferocytosis and inflammation resolution in stroke mice
}

\author{
Wei Cai, ${ }^{1}$ Xuejiao Dai, ${ }^{1}$ Jie Chen, ${ }^{1,2}$ Jingyan Zhao, ${ }^{1}$ Mingyue Xu, ${ }^{1}$ Lili Zhang, ${ }^{1}$ Boyu Yang, ${ }^{1}$ \\ Wenting Zhang, ${ }^{1}$ Marcelo Rocha, ${ }^{1}$ Toshimasa Nakao, ${ }^{3}$ Julia Kofler, ${ }^{4}$ Yejie Shi, ${ }^{1,2}$ R. Anne Stetler, ${ }^{1}$ \\ Xiaoming $\mathrm{Hu},{ }^{1,2}$ and Jun Chen ${ }^{1,2}$ \\ 'Pittsburgh Institute of Brain Disorders and Recovery, and Department of Neurology, University of Pittsburgh, Pittsburgh, \\ Pennsylvania, USA. Ceriatric Research, Education and Clinical Center, Veterans Affairs Pittsburgh Health Care System, \\ Pittsburgh, Pennsylvania, USA. ${ }^{3}$ T.E. Starzl Transplantation Institute and Department of Immunology, University of \\ Pittsburgh, Pittsburgh, Pennsylvania, USA. ${ }^{4}$ Department of Pathology, University of Pittsburgh Medical Center, Pittsburgh, \\ Pennsylvania, USA.
}

Efferocytosis, or phagocytic clearance of dead/dying cells by brain-resident microglia and/or infiltrating macrophages, is instrumental for inflammation resolution and restoration of brain homeostasis after stroke. Here, we identify the signal transducer and activator of transcription 6 / arginase1 (STAT6/Arg1) signaling axis as a potentially novel mechanism that orchestrates microglia/ macrophage responses in the ischemic brain. Activation of STAT6 was observed in microglia/ macrophages in the ischemic territory in a mouse model of stroke and in stroke patients. STAT6 deficiency resulted in reduced clearance of dead/dying neurons, increased inflammatory gene signature in microglia/macrophages, and enlarged infarct volume early after experimental stroke. All of these pathological changes culminated in an increased brain tissue loss and exacerbated longterm functional deficits. Combined in vivo analyses using BM chimeras and in vitro experiments using microglia/macrophage-neuron cocultures confirmed that STAT6 activation in both microglia and macrophages was essential for neuroprotection. Adoptive transfer of WT macrophages into STAT6-KO mice reduced accumulation of dead neurons in the ischemic territory and ameliorated brain infarction. Furthermore, decreased expression of Arg1 in STAT6-/- microglia/macrophages was responsible for impairments in efferocytosis and loss of antiinflammatory modality. Our study suggests that efferocytosis via STAT6/Arg1 modulates microglia/macrophage phenotype, accelerates inflammation resolution, and improves stroke outcomes.

Authorship note: WC and XD contributed equally to this work.

Conflict of interest: The authors have declared that no conflict of interest exists.

Copyright: (c) 2019, American Society for Clinical Investigation.

Submitted: June 27, 2019

Accepted: September 11, 2019

Published: October 17, 2019.

Reference information: JCI Insight. 2019;4(20):e131355.

https://doi.org/10.1172/jci.

insight.131355.

\section{Introduction}

Ischemic stroke results in a wave of neuronal death, starting from the core of blood flow reduction and spreading, if uncontrolled, toward the peri-infarct area over time. Such acute-stage cell death leads to rapid inflammatory responses that could help limit tissue injury. These inflammatory episodes, however, should be well controlled and properly terminated to avoid bystander damage and to allow a permissive environment for tissue repair or regeneration. Failure in inflammation resolution has been implicated in progressive brain damage and long-term neurological deficits after stroke (1). Persistent confrontation with dead or dying cells or cell debris is a major impetus for detrimental inflammatory states $(2,3)$. Therefore, effective efferocytosis, or the phagocytic clearance of dead/dying cells by brain-resident microglia and/or infiltrated macrophages, minimizes the expansion of neural inflammation and restores brain homeostasis (4-6), thereby preventing further cell death.

Both microglia and macrophages are highly plastic cells and are able to change their functional status in response to different external cues. The so-called proinflammatory microglia/macrophage state leads to the release of detrimental factors that evokes neuroinflammation and impedes poststroke brain repair. In contrast, the antiinflammatory microglia/macrophage state facilitates resolution of local inflammation and release of trophic factors that promote tissue repair (7). Accumulating evidence suggests that the process of efferocytosis may resolve inflammation through a reciprocal interaction between the dead/dying cells and the phagocytes (8). Phagocytes physically sequester dead or dying cells to reduce the release of 
damage-associated molecular patterns and are reprogrammed into an antiinflammatory phenotype (9). However, the precise signaling mechanisms that regulate the phagocytic activity of microglia/macrophages in the ischemic brains and dictate their phenotype shift after efferocytosis are currently elusive.

Several members of the STAT family are involved in regulating microglia/macrophage functional status (7). In particular, STAT6 signaling appears to induces the antiinflammatory phenotype of macrophages (10), while the activation of STAT1 facilitates proinflammatory activities (11). Moreover, IL-4, a STAT6 activator, drives microglia/macrophages toward a beneficial phenotype and promotes stroke recovery (12). It remains unknown, however, whether STAT6 plays an indispensable or redundant role in microglia/macrophage phenotypic alterations after stroke, and even less is known about the mechanism whereby STAT6 regulates microglia/macrophage activities in response to ischemic brain damage.

In this study, we discovered that STAT6 was activated in microglia/macrophages in stroke patients. STAT6 signaling defines an antiinflammatory modality of microglia/macrophages in a mouse model of ischemic stroke induced by transient middle cerebral artery (MCA) occlusion (tMCAO). STAT6 deficiency resulted in a shift of microglia/macrophages toward an adverse phenotype, along with impaired clearance of dead/dying neurons and augmented cerebral inflammation and neuronal death. In vitro studies confirmed that STAT6-KO in microglia/macrophages led to impaired efferocytic activities and exacerbated inflammatory responses when cocultured with ischemia-injured neurons. Decreased arginase 1 (Arg1) expression in $S T A T 6^{-1-}$ microglia/macrophages was, at least in part, responsible for their phagocytic dysfunction. These observations, thus, identify the STAT6/Arg1 axis as an important signaling mechanism for microglia/macrophage phenotypic regulation in the context of ischemic stroke and as a viable target for stroke treatment.

\section{Results}

STAT6 is activated in microglia/macrophages in a mouse model of stroke and in stroke patients. The temporal and spatial profiles of STAT6 activation in the peri-infarct areas were assessed in mouse brains after tMCAO (Figure 1A) using an antibody that specifically recognizes the active phosphorylated form of STAT6 (pSTAT6, Tyrosine 641, Supplemental Figure 1A; supplemental material available online with this article; https://doi. org/10.1172/jci.insight.131355DS1). Staining for pSTAT6 was not detectable in sham brains (Supplemental Figure 1A) and was only weakly expressed 1 day after ischemia (Figure 1B). An increase in pSTAT6 was observed in Iba $1^{+}$microglia/macrophages from 3-7 days (3d-7d) after tMCAO (Figure 1, B-D). STAT6 is imported from the cytosol into the nucleus after activation (13). In Figure 1C, a 3-dimensional-reconstructed (3-D-reconstructed) confocal image shows the localization of pSTAT6 within the nucleus of an Iba1 ${ }^{+}$ cell. No obvious pSTAT6 staining was observed in other CNS cells, including $\mathrm{NeuN}^{+}$neurons, $\mathrm{GFAP}^{+}$astrocytes, $\mathrm{APC}^{+}$oligodendrocytes, or $\mathrm{CD} 31^{+}$endothelial cells after stroke (Supplemental Figure 1B).

Flow cytometry analysis confirmed the cellular specificity of pSTAT6 staining in the ischemic brain 3d after stroke induction. Cell-specific marker combinations were used to identify CD $45^{+} \mathrm{CD} 11 \mathrm{~b}^{+}$microglia/macrophages, CD45-GLAST ${ }^{+}$astrocytes, $\mathrm{CD}^{-} 5^{-} \mathrm{O}^{+}$oligodendrocytes, and $\mathrm{CD}^{-} 5^{-} \mathrm{NeuN}^{+}$neurons (Figure 1E). Consistent with the immunohistological staining, pSTAT6 was strongly expressed in $\mathrm{CD}_{4} 5^{+} \mathrm{CD} 11 \mathrm{~b}^{+}$microglia/ macrophages but was barely detectable in other CNS cells (Figure 1F). We further divided the CD $45^{+} \mathrm{CD} 11 \mathrm{~b}^{+}$ population into $\mathrm{CD} 45^{\text {intermediate }} \mathrm{CD} 11 \mathrm{~b}^{+}$microglia and $\mathrm{CD} 45^{\text {hi }} \mathrm{CD} 11 \mathrm{~b}^{+}$macrophages (Figure $\left.1 \mathrm{G}\right)$. pSTAT6 signal could be detected in both subpopulations. The $\mathrm{CD} 45^{\text {hi }} \mathrm{CD} 11 \mathrm{~b}^{+}$population showed higher pSTAT6 expression as determined by mean fluorescence intensity (MFI) (Figure 1, G and $\mathrm{H}$ ).

We then investigated whether STAT6 is activated in human postmortem brain tissue from stroke patients. Expression of pSTAT6 was observed in $\mathrm{Iba1}^{+}$microglia/macrophages in the ischemic brain areas in stroke patients, while less pSTAT6/Iba1 colocalization was observed in nonstroke brain (Supplemental Figure 2, A-C). The pSTAT6 staining partially overlapped with DAPI nuclei staining (Supplemental Figure 2, C-E). The pSTAT6 signal was also noted in some Iba1- cells in both nonstroke and stroke brains, consistent with a previous report suggesting STAT6 activation in other types of cells in diseased or aging human brains (14).

STAT6 deficiency exacerbates brain infarction and neuronal death after $1 M C A O$. Male C57BL/6J WT mice and STAT6-KO mice on a C57BL/6J background were used to assess the influence of STAT6 activation on stroke outcomes. Regional cortical cerebral blood flow (rCBF) was monitored before ischemia, during ischemia, and 15 minutes after reperfusion. $\mathrm{CCBF}$ was similar between WT and STAT6-KO mice throughout the surgery and during reperfusion (Figure 2A), suggesting a comparable extent of ischemic insult and recovery of blood flow between these 2 lines. Compared with WT mice, infarct volume was significantly increased in STAT6-KO mice 3d and 7d after tMCAO (Figure 2B). 
A

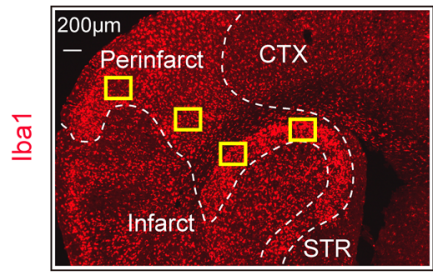

B
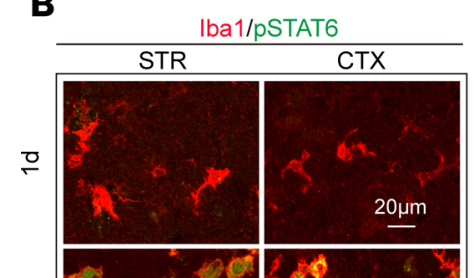

ரे

융
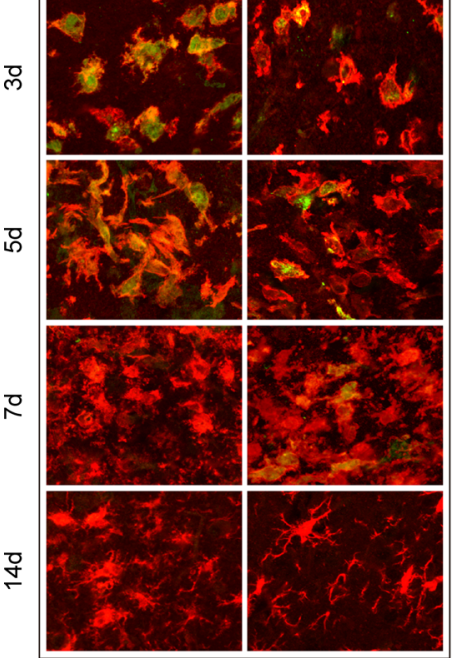

C

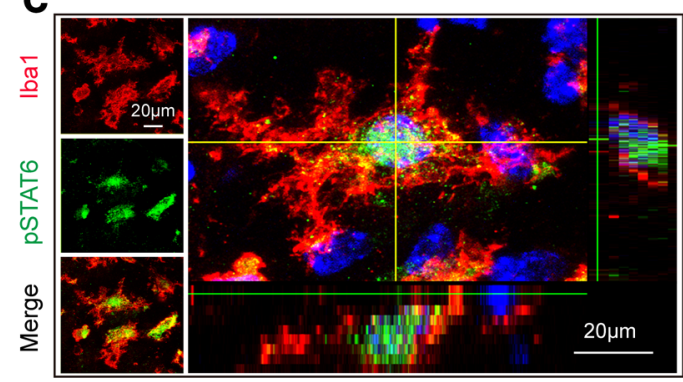

E
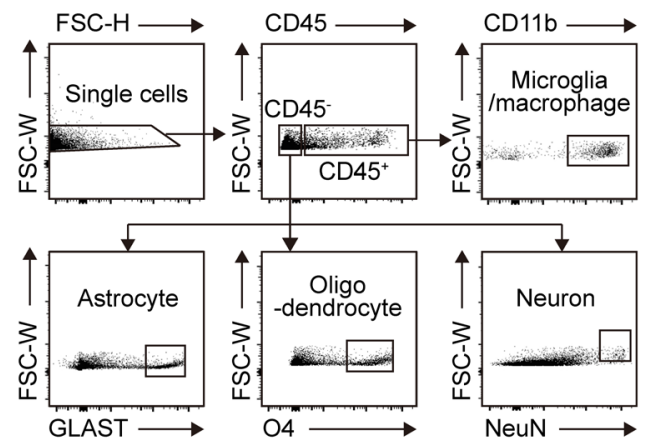
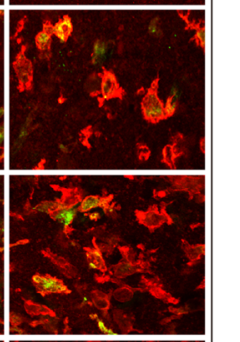

\section{G}

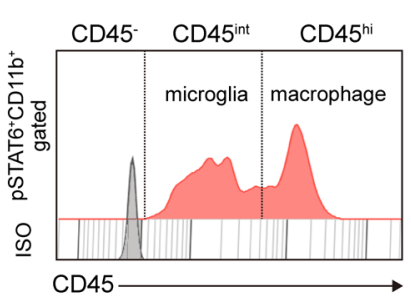

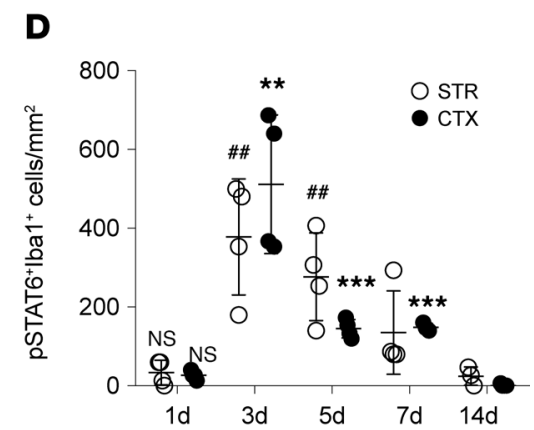

F

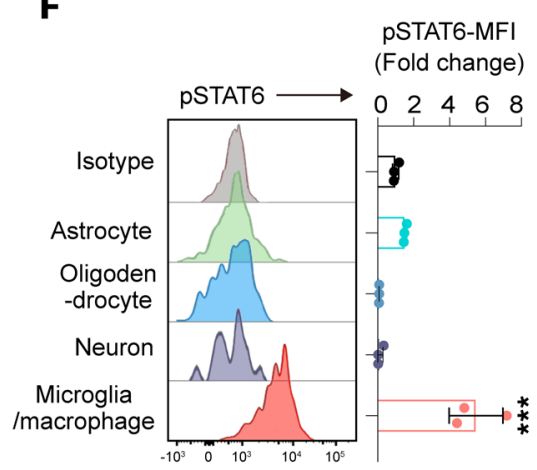

H

Figure 1. STAT6 is activated in microglia/macrophages after tMCAO. (A) Representative image demonstrates the peri-infarct area defined by lba1 staining. Two randomly selected microscopic fields in the cortex (CTX) and 2 in the striatum (STR) of each section were subjected for analysis. Scale bar: $200 \mu \mathrm{m}$. (B) Representative images of coronal brain sections showing the phosphorylation of STAT6 (green) in Iba $1^{+}$microglia/macrophages (red) at indicated time points after tMCAO. Scale bar: $20 \mu \mathrm{m}$. (C) Representative images of coronal brain slices collected 3d after tMCAO showing the nuclear (DAPI, blue) localization of pSTAT6 (green) in Iba1 ${ }^{+}$(red) microglia/macrophages. Scale bar: $20 \mu \mathrm{m}$. (D) Quantification of the number of pSTAT6 ${ }^{+}$lba1 ${ }^{+}$cells in the ischemic regions indicated in $\mathbf{B}$ at different time points after tMCAO. $n=3-4$ mice per group. ${ }^{* *} P \leq 0.01,{ }^{* * *} P \leq 0.001 \mathrm{vs}$. 1d in CTX. \#\# $P \leq 0.01$ vs. 1d in STR, 1-way ANOVA. (E and $\mathbf{F}$ ) Flow cytometric analysis of pSTAT6 in brain cells $3 \mathrm{~d}$ after tMCAO. (E) Representative dot plots demonstrate the gating strategy for microglia/macrophages

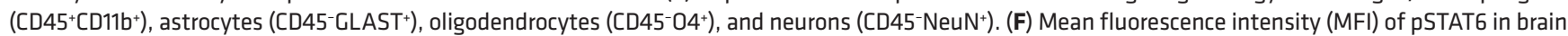
cells. $n=3$ mice. ${ }^{* * *} P \leq 0.01$, Student's $t$ test. (C) Representative plot of STAT6 activation (pSTAT6) in microglia (CD45 intermediate; CD45int) and macrophages $\left(\mathrm{CD}^{\mathrm{h}} 5^{\text {hi}}\right)$ in pSTAT6 ${ }^{+} \mathrm{CD11}^{+}$population. (H) STAT6 activation evaluation in microglia and macrophages by MFI of pSTAT6. $n=3$ mice. ${ }^{* *} P \leq 0.01$, Student's $t$ test.

Consistent with the enlargement of brain infarction, more dead or dying neurons $\left(\mathrm{NeuN}^{+} \mathrm{TUNEL}^{+}\right.$ cells) were observed in male STAT6-KO mice compared with male WT mice in the peri-infarct regions (a region approximately 300-400 $\mu \mathrm{m}$ wide, surrounding the border of stroke infarct) in both striatum and cortex $3 \mathrm{~d}$ after stroke (Figure 2, C and D, and Supplemental Figure 3). $\mathrm{NeuN}^{+}$cells were barely detected in the ischemic core. The number of dead/dying neurons were reduced in WT mice $7 \mathrm{~d}$ after stroke. In contrast, more dead/dying neurons remained in STAT6-KO mice, especially in the ischemic cortex at $7 \mathrm{~d}$ after $\mathrm{tMCAO}$. The reduction rate of dead/dying neurons in the cortex and striatum from $3 \mathrm{~d}-7 \mathrm{~d}$ after stroke was lower in STAT6-KO mice as compared with WT mice (Figure 2E).

Sex differences in response to stroke and stroke treatment has been well documented (15). The risk and severity of stroke increase in females after menopause. Ovariectomized (OVX) female mice were therefore used to induce estrogen decline, simulating the postmenopausal state in order to test the effect of STAT6 on stroke outcomes in at-risk females. Brain infarction and neurological deficits was exacerbated in OVX STAT6-KO female mice 3d after tMCAO (Figure 3).

The phagocytic clearance of dead/dying neurons was then determined by detecting the appearance of $\mathrm{NeuN}^{+}$(neuronal) material within Iba1 ${ }^{+}$microglia/macrophages in the peri-infarct area (Figure 4, A and B). 
A WT STAT6 KO

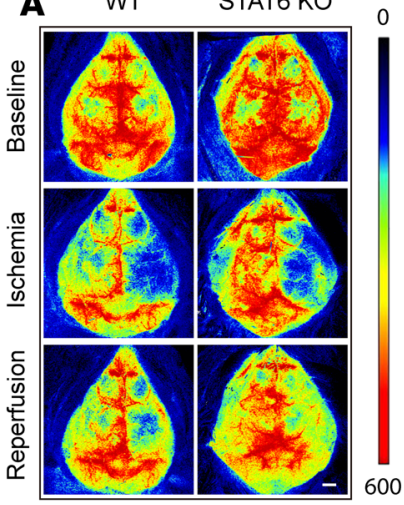

C

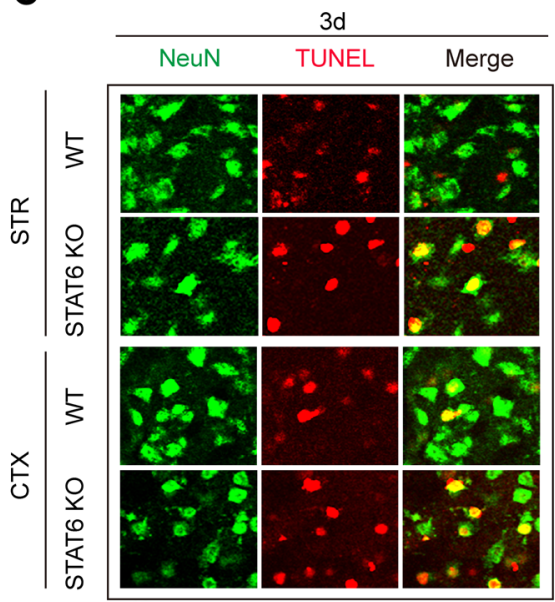

- WT - STAT6 KO

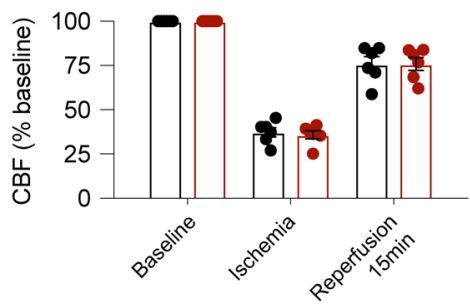

B
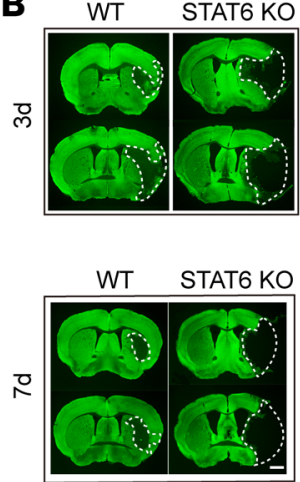

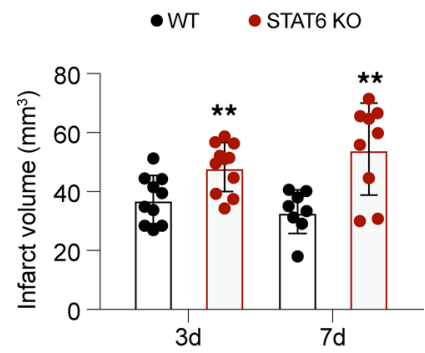

- WT - STAT6 KO

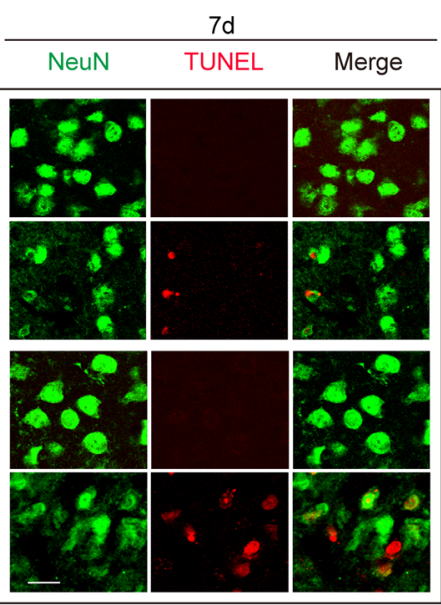

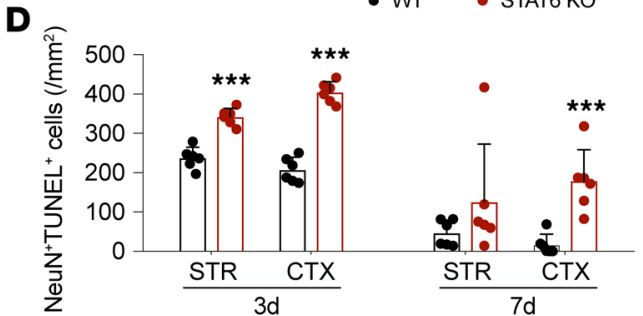

$\mathbf{E}$

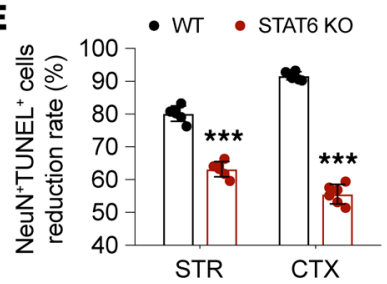

Figure 2. STAT6 deficiency exacerbates brain infarction and neuronal death after tMCAO. Male WT and STAT6-KO mice were subjected to 60 minutes of tMCAO. (A) No difference in regional cerebral blood flow (CBF) between WT and STAT6-KO mice was detected. $n=6$ mice per group. Scale bar: $1 \mathrm{~mm}$. (B) Infarct volume at $3 d(n=10-11$ mice per group) and $7 d(n=8-9$ mice per group) after tMCAO was quantified in MAP2-stained (green) coronal sections. Dashed lines outline the infarct area. Scale bar: $1 \mathrm{~mm}$. (C-E) Neuronal death increased in STAT6-KO brains 3d and 7d after tMCAO. (C) Representative images demonstrating TUNEL (red) colabeling with the neuronal marker NeuN (green) in infarct areas indicated in Figure 1A. Scale bar: $10 \mu \mathrm{m}$. (D) Quantification of NeuN ${ }^{+}$TUNEL $^{+}$neurons in cortex (CTX) and striatum (STR). (E) The reduction rate of dead/dying neurons from 3d-7d after tMCAO was calculated as described in Methods. $n=6$ mice per group. ${ }^{* *} P \leq 0.01,{ }^{* * *} P \leq 0.001$ STAT6-KO vs. WT, Student's $t$ test.

Although the total numbers of $\mathrm{Iba}^{+}$microglia/macrophages were similar between the 2 genotypes (Figure $4 \mathrm{C}$ ), the number of $\mathrm{Iba}^{+} \mathrm{NeuN}^{+}$cells, indicating phagocytic microglia/macrophages, was reduced in STAT6KO mice $3 \mathrm{~d}$ after stroke compared with WT mice (Figure 4D). No Iba $1^{+} \mathrm{NeuN}^{+}$cells were observed in WT or STAT6-KO sham nonischemic brains (Supplemental Figure 5D). Quantification of the triple-staining of Iba1/TUNEL/NeuN further confirmed reduced phagocytosis of dead/dying neurons by microglia/macrophages $\left(\mathrm{Iba}^{+} \mathrm{NeuN}^{+} \mathrm{TUNEL}^{+}\right.$, Figure 4E) in STAT6-KO mice, resulting in an accumulation of uncleared dead/dying neurons ( $\mathrm{Iba1}^{-} \mathrm{NeuN}^{+} \mathrm{TUNEL}^{+}$, Figure $4 \mathrm{~F}$ ). As a result, the phagocytic index, which was calculated as the proportion of dead/dying neurons engulfed by microglia/macrophages, was lower in STAT6-KO mice (Figure 4G). Very few Iba1 ${ }^{+} \mathrm{NeuN}^{+} \mathrm{TUNEL}^{-}$cells were observed in the peri-infarct area in both WT and STAT6-KO brain (Figure 4H). Taken together, these data suggest that STAT6 deficiency may impair microglia/macrophage-mediated clearance of dead/dying neurons in ischemic brains.

STAT6 deficiency potentiates proinflammatory microglia/macrophage responses and aggravates poststroke inflammation. Since phosphorylated (activated) STAT6 was observed mainly in microglia/macrophages after ischemic stroke (Figure 1), we wished to determine the effects of STAT6-KO on microglia/macrophage responses in the ischemic brain. We found that STAT6-KO elevated the numbers of $\mathrm{Iba} 1^{+} \mathrm{CD} 16^{+}$proinflammatory microglia/ macrophages and reduced $\mathrm{Iba}^{+} \mathrm{CD} 206^{+}$antiinflammatory phagocytes at both $3 \mathrm{~d}$ and $7 \mathrm{~d}$ after $\mathrm{MCAO}$ (Figure 5, A-D, and Supplemental Figure 4). To further confirm the association of STAT6 with microglia/macrophage phenotypic changes, we collected ischemic brains from WT mice $3 \mathrm{~d}$ after tMCAO for flow cytometry analysis. 


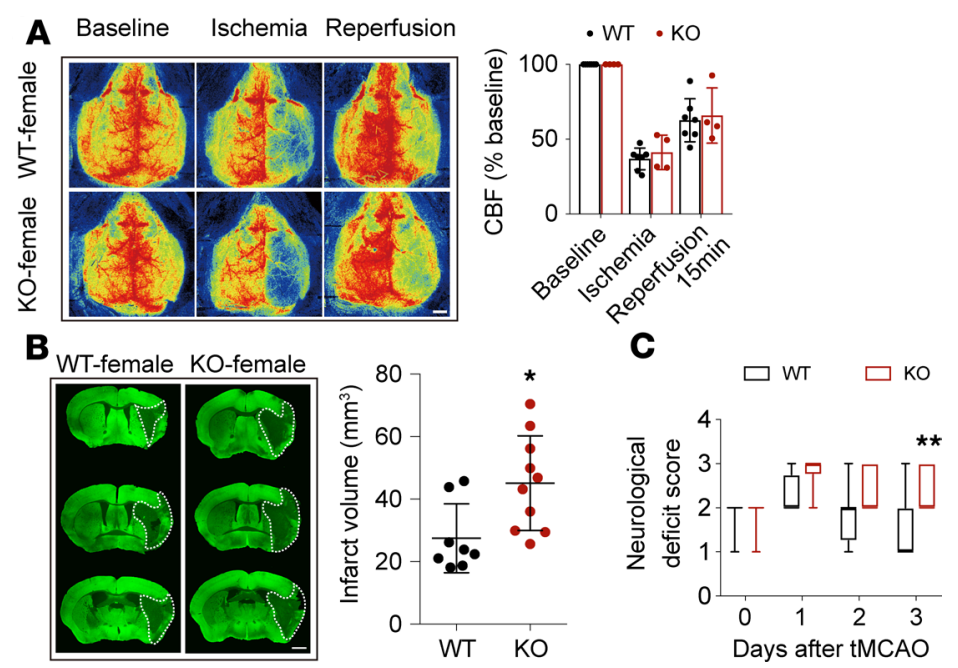

Figure 3. STAT6 deficiency exacerbates brain infarction in ovariectomized female mice after tMCAO. (A) There was no difference in cerebral blood flow (CBF) between female WT and STAT6-KO mice. $n=7$ for WT and $n=4$ for STAT6-KO. (B) Infarct volume $3 \mathrm{~d}$ after tMCAO was quantified on MAP2-stained (green) coronal sections. Dashed lines define the infarct area. $n=8$ for WT and $n=10$ for STAT6-KO. Scale bar: $1 \mathrm{~mm}$. (C) Neurological deficit score was assessed during the first 3d after tMCAO. $n=8$ for WT and $n=10$ for STAT6-KO. ${ }^{*} P \leq 0.05,{ }^{* *} P \leq 0.01 \mathrm{vs}$. WT, Student's $t$ test (A and B) or Mann-Whitney $U$ test (C).

Microglia/macrophages expressing pSTAT6 $\left(\mathrm{pSTAT} 6{ }^{+} \mathrm{CD} 45^{+} \mathrm{CD} 11 \mathrm{~b}^{+}\right)$displayed stronger mean fluorescent intensity (MFI) of CD206 and weaker MFI of CD16 as compared with the subset of microglia/macrophages from the same samples that did not express pSTAT6 (pSTAT6- $\mathrm{CD}^{4} 5^{+} \mathrm{CD} 11 \mathrm{~b}^{+}$, Figure 5E).

Consistent with a phenotypic shift toward a proinflammatory modality, STAT6 deficiency led to augmented local inflammation in the ischemic brain, despite not altering the state of the immune milieu in sham animals (Supplemental Figure 5A). The mRNA expression of proinflammatory cytokines, including IL- $1 \alpha$, IL- 6 , and TNF- $\alpha$, and some markers for the proinflammatory phenotypes (CD16 and CD86), increased at $3 \mathrm{~d}$ and/or $7 \mathrm{~d}$ after stroke in STAT6-KO brains as compared with WT. In contrast, the mRNA expression of antiinflammatory cytokines (IL-10 and IL-4) and markers for antiinflammatory phenotypes (Arg1 and CD206) decreased in the STAT6-KO ischemic brain (Figure 5F).

We did not observe significant differences between genotypes in the numbers of $\mathrm{CD} 45^{\text {intermediate }} \mathrm{CD} 11 \mathrm{~b}^{+}$ microglia or $\mathrm{CD} 45^{\text {hi }} \mathrm{CD} 11 \mathrm{~b}^{+}$macrophages in the ischemic brain (Supplemental Figure $6 \mathrm{~A}$ ). There were also no significant differences in the numbers of other types of peripheral immune cells, including $\mathrm{CD} 45^{+} \mathrm{F} 4 / 80^{+}$ monocytes/macrophages, $\mathrm{CD} 45^{+} \mathrm{Ly}_{6 \mathrm{G}}{ }^{+}$neutrophils, $\mathrm{CD} 45^{+} \mathrm{CD} 3^{+} \mathrm{T}$ cells, and $\mathrm{CD} 45^{+} \mathrm{CD} 19^{+} \mathrm{B}$ cells in the blood and spleen 3d after tMCAO (Supplemental Figure 6, B-D). These observations indicate that the differences in response to stroke between genotypes likely were not a result of global changes in the cellular components of the immune system.

Both CNS microglia and peripheral macrophages contribute to STAT6-afforded protection against ischemic brain injury. To determine whether the observed increase in STAT6 signaling occurred in microglia or macrophages, or both, after stroke, we used chimeric mice derived by transferring the BM of CX3CR1GFP mice to irradiated WT mice (Figure 6A). The chimera enabled us to distinguish infiltrating GFP ${ }^{+}$ macrophages among the $\mathrm{CD} 45^{+} \mathrm{CD} 11 \mathrm{~b}^{+}$populations (including microglia, macrophages, and neutrophils) in the ischemic brain. The appearance of $\mathrm{GFP}^{+}$cells in the ischemic brain $3 \mathrm{~d}$ after stroke confirmed the success of hematopoietic cell reconstitution (Figure 6B). Image stream analysis showed that pSTAT6 was detected in both $\mathrm{CD} 45^{+} \mathrm{CD} 11 \mathrm{~b}^{+} \mathrm{GFP}^{+}$macrophages and $\mathrm{CD} 45^{+} \mathrm{CD} 11 \mathrm{~b}^{+} \mathrm{GFP}^{-}$cells (Figure $6, \mathrm{~B}$ and $\mathrm{C}$, and Supplemental Figure 7). Interestingly, the majority of $\mathrm{GFP}^{+}$macrophages were pSTAT $^{+}$, whereas only $\sim 31 \%$ of GFP $^{-}$cells, mainly microglia, were pSTAT $^{+} 3 \mathrm{~d}$ after tMCAO (Figure 6D). Arg1 was expressed in both $\mathrm{CD} 45^{+} \mathrm{CD} 11 \mathrm{~b}^{+} \mathrm{GFP}^{+}$macrophages and $\mathrm{CD} 45^{+} \mathrm{CD} 11 \mathrm{~b}^{+} \mathrm{GFP}{ }^{-}$cells in the ischemic brain $3 \mathrm{~d}$ after stroke (Figure 6D).

We next constructed different chimeras to confirm whether CNS STAT6 or peripheral STAT6 is important for stroke outcomes (Figure 6E). Three types of chimeras were studied: (a) WT BM transferred into WT recipients (WT/WT); (b) STAT6-KO BM transferred to WT recipients (WT/KO); and (c) WT BM 
A

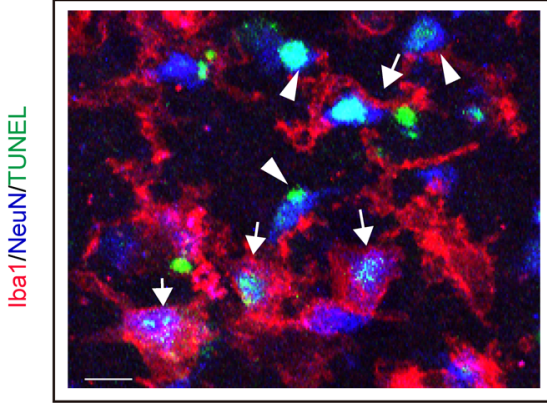

$$
\text { - WT KO }
$$
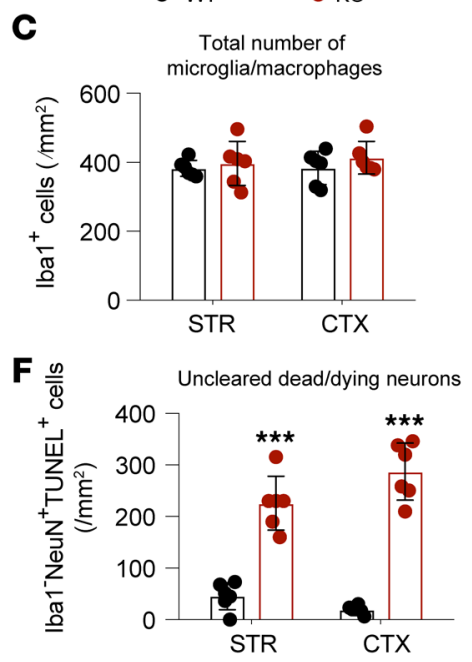

STAT6 KO

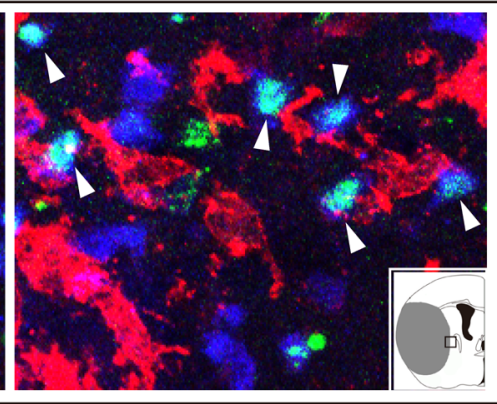

D
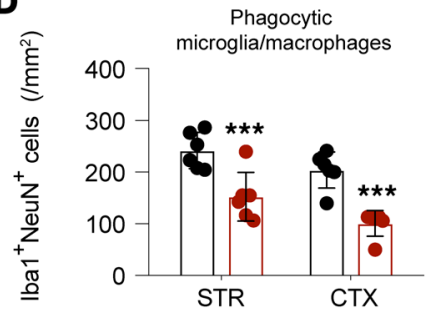

G

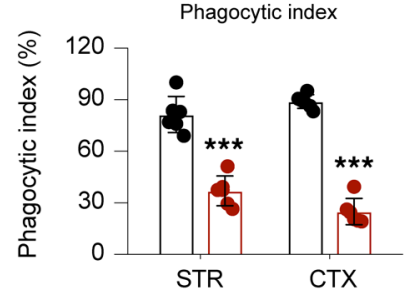

B

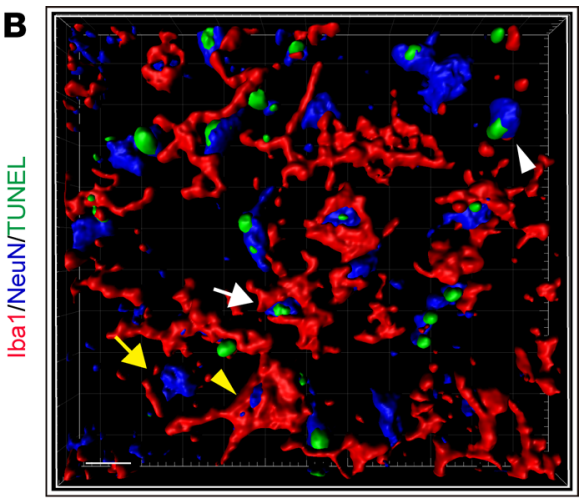

E

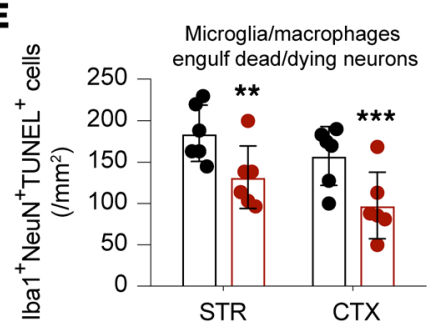

H

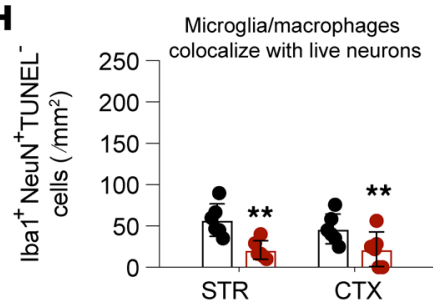

Figure 4. Capacity of dead/dying neuron clearance in microglia/macrophages was impaired in STAT6-KO mice. WT and STAT6-KO mice were subjected to 60 minutes of tMCAO. Brains were collected 3d after tMCAO. (A) Representative images of NeuN (blue), TUNEL (green), and Iba1 (red) triple-staining. Scale bar: $10 \mu \mathrm{m}$. The inset image depicts the hemisphere ipsilateral to stroke, where brain infarction is shown in gray and the area for image analysis is indicated by the box. (B) High-power 3-D image generated from $\mathbf{A}$. White arrows indicate microglia/macrophages that engulfed dead/dying neurons ( $\left(\mathrm{ba} 1^{+} \mathrm{NeuN}^{+} \mathrm{TUNEL}^{+}\right.$). White arrowheads indicate dead/dying neurons that were not engulfed by microglia/macrophages (Iba1-NeuN ${ }^{+} \mathrm{TUNEL}^{+}$). Yellow arrows indicate live neurons (Iba1-NeuN+TUNEL-). Yellow arrowhead indicates a TUNEL- neuron touched by an Iba1 ${ }^{+}$cell (Iba1 $\left.1^{+} \mathrm{NeuN}^{+} \mathrm{TUNEL}^{-}\right)$. Scale bar: $10 \mu \mathrm{m}$. (C) Quantification of the total number of Iba1 $1^{+}$microglia/macrophages in ischemic areas as indicated in Figure1A. (D) The number of Iba1 ${ }^{+} \mathrm{NeuN}^{+}$

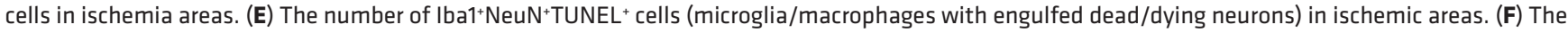
number of Iba1-NeuN ${ }^{+}$TUNEL ${ }^{+}$nonengulfed dead neurons in ischemic areas was quantified. (G) Phagocytic index, the percentage of dead/dying neurons

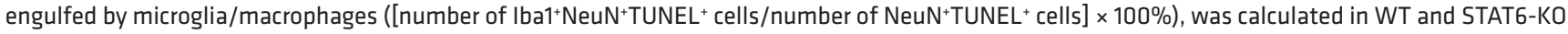
brains. (H) Quantification of $\mathrm{Iba}^{+} \mathrm{NeuN}^{+} \mathrm{TUNEL}^{-}$cells (microglia/macrophages colocalize with TUNEL- neurons) in ischemic areas. $n=6$ mice per group. ${ }^{* *} P$ $\leq 0.01,{ }^{* *} P \leq 0.001$ STAT6-KO vs. WT, Student's $t$ test.

transferred to STAT6-KO recipients (KO/WT). The absence of STAT6 in either the CNS (KO/WT) or the periphery (WT/KO) worsened stroke outcomes, as indicated by enlarged infarct volume (Figure 6, F and $\mathrm{G}$ ), and increased numbers of dead/dying neurons ( $\mathrm{NeuN}^{+} \mathrm{TUNEL}^{+}$) in the ischemic brain (Figure 6, $\mathrm{H}$ and I) as compared with WT/WT mice at $3 \mathrm{~d}$ after tMCAO. These results suggest that STAT6 activation in both CNS microglia and peripheral cells contributes to STAT6-afforded protection against ischemic brain injury.

It has been reported that i.v. transferred monocyte/macrophages are capable of entering into the ischemic brain and exerting neuroprotection (16). To further confirm the importance of STAT6 in the macrophage population, we adoptively transferred 2 million WT macrophages or STAT6-KO macrophages into STAT6-KO mice 2 hours after tMCAO (Figure 7A). WT macrophage-infused animals displayed reduced brain infarction (Figure 7, B and C) and declined number of dead/dying neurons (NeuN ${ }^{+} \mathrm{TUNEL}^{+}$) in the ischemic brain (Figure 7, D and E) compared with the STAT6-KO macrophage-infused mice $3 \mathrm{~d}$ after stroke.

STAT6 facilitates efferocytosis of dead/dying neurons by microglia and macrophages. We further evaluated the role of STAT6 signaling on the efferocytic activity of microglia and macrophages upon encountering dead/dying neurons in in vitro cell cultures. Primary cortical neurons were exposed to 90 minutes of oxygen-glucose deprivation (OGD), an in vitro model that simulates ischemic injury. Propidium iodide 
A

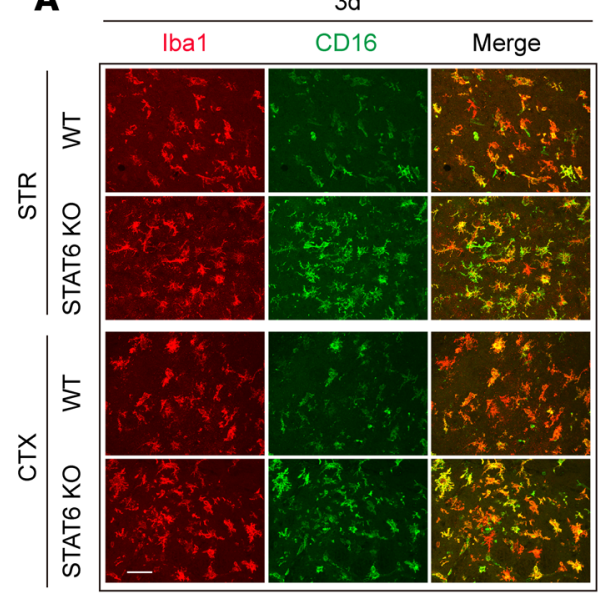

E

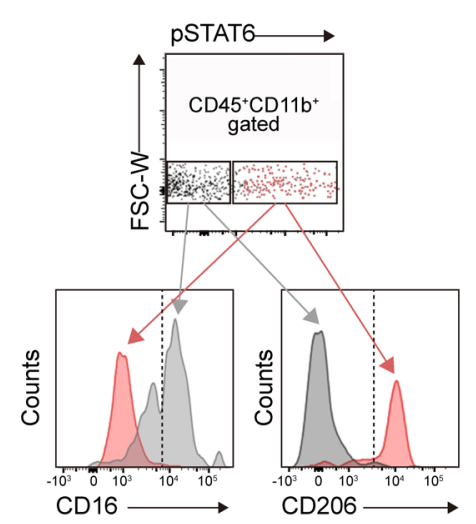

B

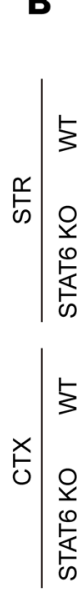

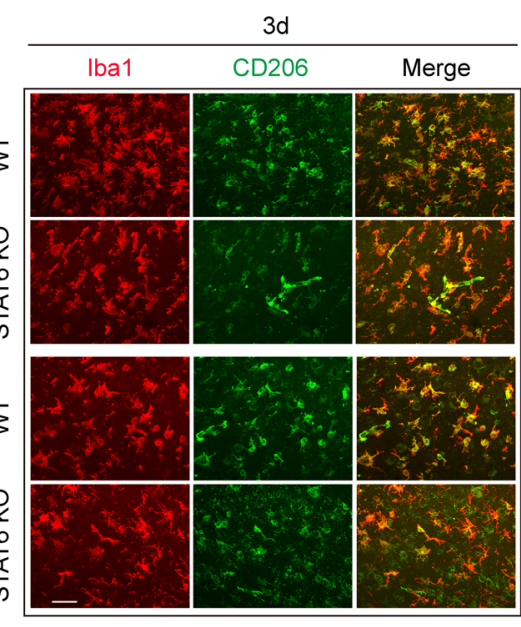

$\mathbf{F}$

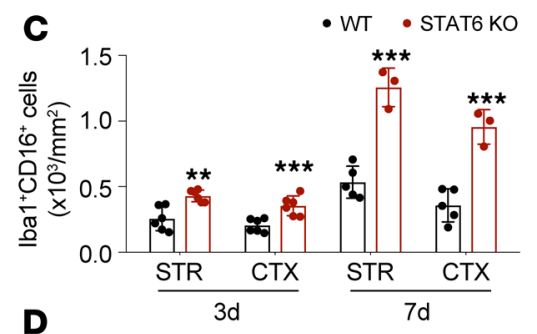

D

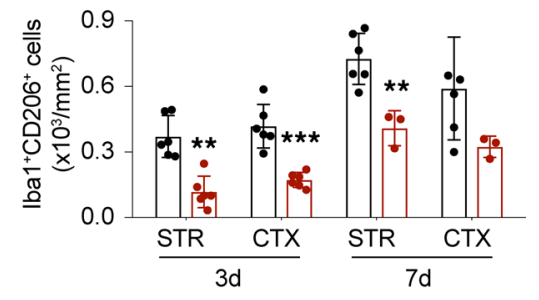

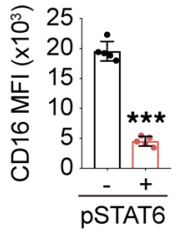
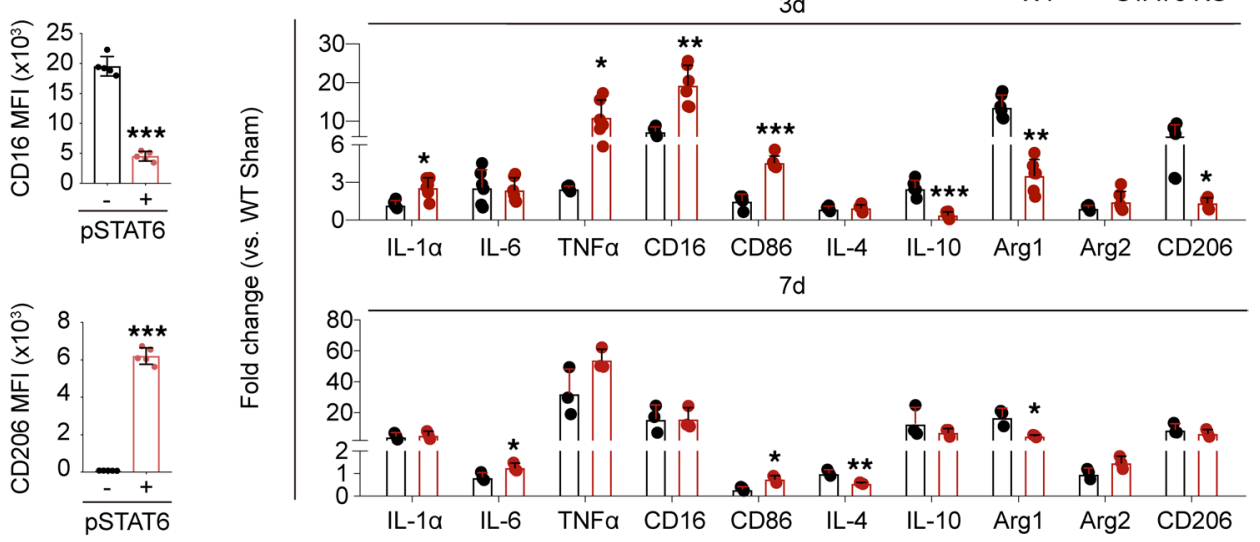

Figure 5. STAT6 deficiency enhances the proinflammatory phenotype of microglia/macrophages and aggravates neuroinflammation after ischemic stroke. Brains were collected from WT and STAT6-KO mice 3d and 7d after 60 minutes tMCAO. (A-D) Analysis of microglia/macrophage phenotypes. (A) Representative images of microglia/macrophage marker lba1 (red) and proinflammatory phenotype marker CD16 (green) double-staining. Scale bar: 50 $\mu \mathrm{m}$. (B) Representative images of microglia/macrophage marker lba1 (red) and antiinflammatory phenotype marker CD206 (green) double-staining. Scale bar: $50 \mu \mathrm{m}$. (C) Quantification of Iba1+CD16+ proinflammatory microglia/macrophages in ischemic areas at 3d and 7d after tMCAO. (D) Quantification of Iba1 ${ }^{+} \mathrm{CD}_{206}$ antiinflammatory microglia/macrophages in ischemic areas at $3 \mathrm{~d}$ and $7 \mathrm{~d}$ after tMCAO. $n=3-6$ mice per group. ${ }^{* *} P \leq 0.01,{ }^{* * *} P \leq 0.001 \mathrm{STAT6}$ KO vs. WT, Student's $t$ test. (E) The expression of CD16 and CD206 in pSTAT6 $6^{+}$and pSTAT6- microglia/macrophages (CD45 ${ }^{+}$CD11 $\left.b^{+}\right)$in ipsilateral hemisphere from WT mice was measured by flow cytometry 3d after tMCAO. Mean fluorescent intensity (MFI) of CD16 or CD206 in pSTAT6-CD45+CD11b+ (gray) and pSTAT6 ${ }^{+} C^{2} 45^{+} C^{2} 11 b^{+}$(pink) populations was quantified. $n=5$ per group. ${ }^{* *} P \leq 0.001$ pSTAT6 ${ }^{+}$vs. pSTAT6 ${ }^{-}$population, Student's $t$ test. (F) mRNA expression of proinflammatory and antiinflammatory markers were measured by RT-qPCR in the ipsilateral hemisphere $3 \mathrm{~d}$ and $7 \mathrm{~d}$ after ischemic stroke. $n=6$ mice per group for 3 d. $n=3$ mice per group for $7 d$. ${ }^{*} P \leq 0.05,{ }^{* *} P \leq 0.01,{ }^{* * *} P \leq 0.001$ STAT6-KO vs. WT, Student's $t$ test.

(PI) was added into neurons 24 hours after OGD (before cell fixation) to label dead/dying cells. WT microglia, WT macrophages, STAT6-KO microglia, or STAT6-KO macrophages were exposed to PI-labeled neurons at a ratio of $1: 10$, and engulfment of $\mathrm{PI}^{+}$dead/dying neurons by microglia or macrophages was evaluated over time (Figure 8, A-D, and Supplemental Figure 8, A and B). The viability of WT and STAT6-KO microglia or macrophages was comparable either before or 6 hours after exposure to PI-labeled dead/dying neurons (Supplemental Figure 8C). STAT6-KO microglia displayed reduced overall efferocytic capacity, as the average number of engulfed dead/dying neurons per microglia was lower from 1-4 hours after coculture compared with WT microglia (Figure 8C). Notably, STAT6-KO macrophages only displayed significantly reduced efferocytosis early (0.5-1 hours) after encountering dead/ dying neurons (Figure 8D); at later time points, STAT6-KO cultures had similar numbers of dead/dying neurons per macrophage compared with WT cultures. The phagocytic activity of STAT6-KO microglia toward fluorescent-labeled latex beads was also reduced compared with WT microglia in response to IL-4 stimulation (Supplemental Figure 9), suggesting that the impaired phagocytosis in STAT6-KO microglia might not be specific to dead/dying neurons but reflects an overall reduction of phagocytic capacity of these microglia deficient in STAT6 signaling. 
A
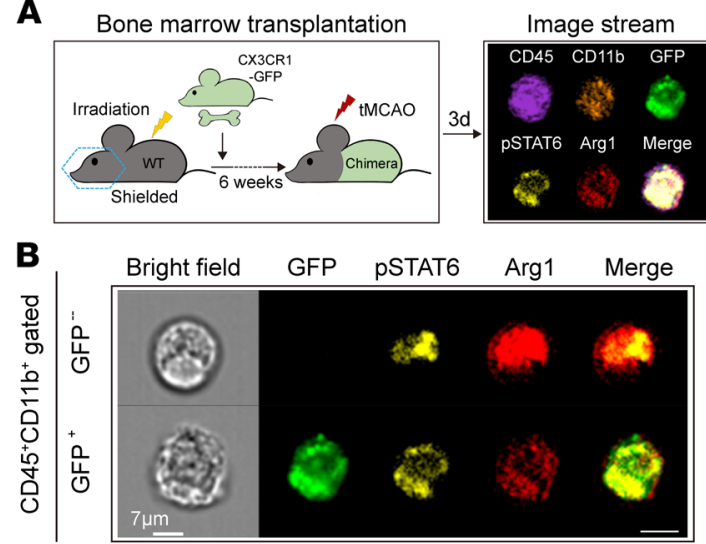

C

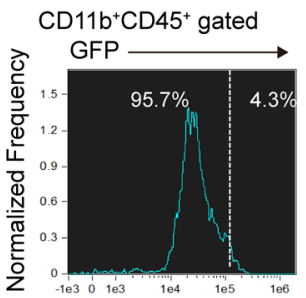

D $\quad \mathrm{CD} 11 \mathrm{~b}^{+} \mathrm{CD} 45^{+}$gated

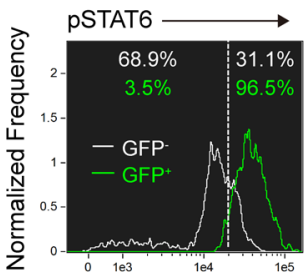

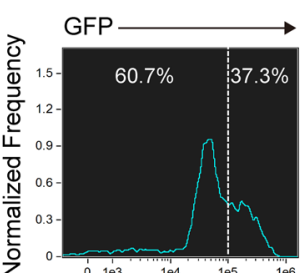

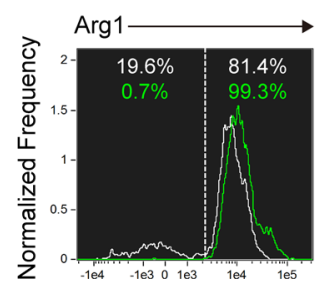

E

Donor Receipient Chimera

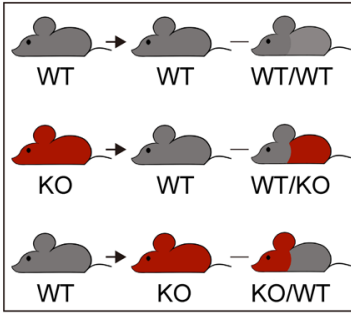

$\mathbf{F}$

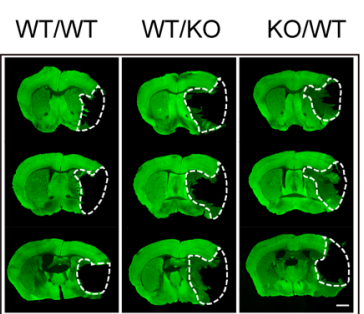

H

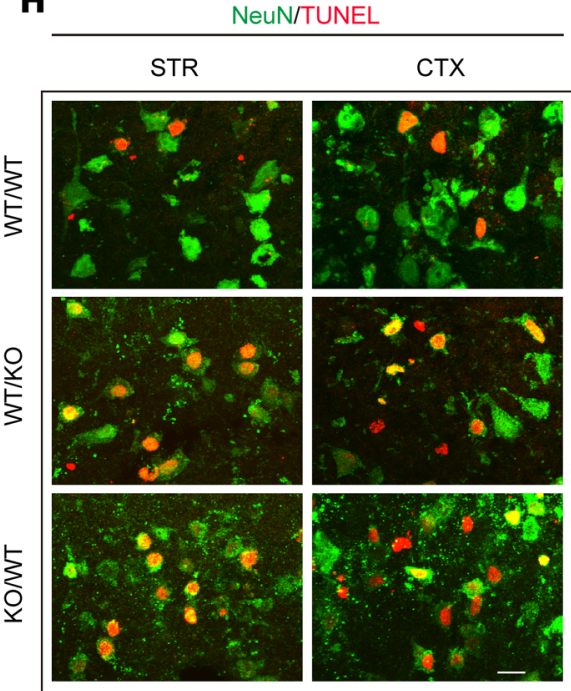

G

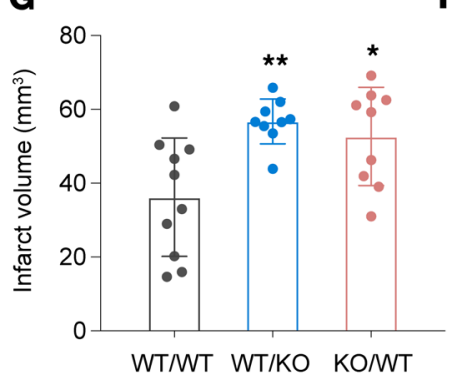

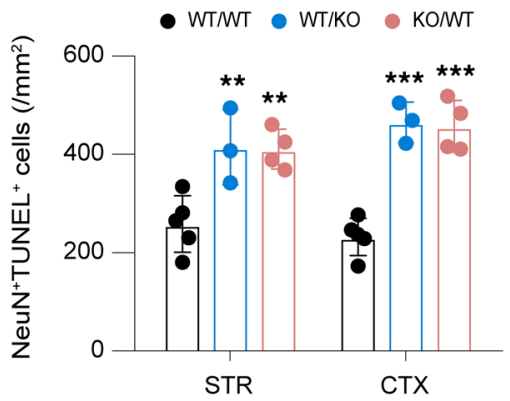

Figure 6. Both CNS microglia and peripheral macrophages contribute to STAT6-afforded protection against ischemic stroke. (A) Schematic illustration for the construction of WT/CX3CR1-GFP chimeric mice. (B-D) ImageStream analysis of pSTAT6 and Arg1 in CD45+CD11b+CFP- cells and CD45+CD11b+GFP+ macrophages in ipsilateral hemisphere of chimeric mice was performed 3d after tMCAO. (B) Representative images indicating pSTAT6 and Arg1 signal in both CD45+CD11b+CFP- cells and CD45+CD11 b+GFP+ macrophages in ipsilateral brains. Scale bar: $7 \mu \mathrm{m}$. (C) Percentages of GFP- cells and GFP+ macrophages among CD45+CD11 b cells were assessed in WT (left) and WT/CX3CR1-GFP chimeric mice (right). (D) Percentages of pSTAT6 ${ }^{+}$cells (left) and Arg1 ${ }^{+}$cells (right) observed among CD45+CD11 b+CFP- cells and CD45+CD11 $\mathrm{b}^{+} \mathrm{CFP} \mathrm{P}^{+}$macrophages. $n=3$ mice. (E) Chimeric mice were constructed by transplanting WT BM to WT recipients (WT/WT), STAT6-KO BM to WT recipients (WT/KO), or WT BM to STAT6-KO recipients (KO/WT) after irradiation of recipients. Chimeric mice were subjected to 60 minutes of tMCAO at 6 weeks after irradiation. (F and $\mathbf{G}$ ) Infarct volume at 3d after tMCAO was quantified in MAP2-stained (green) coronal sections. Dashed lines outline the infarct area. $n=9-10$ mice per group. Scale bar: $1 \mathrm{~mm}$. (H and I) Neuronal death in chimeric mice was quantified by TUNEL (red) and NeuN (green) colabeling in infarct areas $3 \mathrm{~d}$ after tMCAO. Scale bar: $10 \mu \mathrm{m} . n=3-5$ mice per group. ${ }^{*} P \leq 0.05$, ${ }^{* *} P \leq 0.01$, ${ }^{* *} P \leq 0.001$ vs. WT/WT, 1-way ANOVA.

STAT6 deficiency enhances proinflammatory responses in both microglia and macrophages after efferocytosis. We then evaluated whether STAT6 is important for the inflammatory status of microglia or macrophages tied to efferocytosis of dead/dying neurons. The mRNA of the proinflammatory cytokine IL- 6 increased in WT microglia and macrophages exposed to PI-labeled dead/dying neurons for 1 hour (Figure 8E), whereas STAT6 deficiency in either microglia or macrophages resulted in further elevation in IL-6 mRNA. STAT6KO macrophages also exhibited significantly increased TNF- $\alpha$ mRNA and reduced IL-10 mRNA compared with WT macrophages (Figure 8E). We further quantified cytokine production in microglia and macrophages after a 6-hour exposure to PI-labeled dead/dying neurons using flow cytometry. More than $90 \%$ of WT and STAT6-KO microglia and macrophages displayed evidence of phagocytosis in the presence of the PI signal

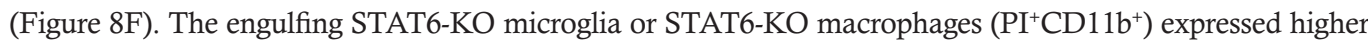
levels of IL- 6 and TNF- $\alpha$, but lower levels of IL-10 as compared with their WT counterparts (Figure 8, F-H). In the absence of stimulation, STAT6-KO microglia/macrophages had comparable basal levels of cytokine expression (Figure 8E and Supplemental Figure 10, A and B). These data suggest that STAT6 signaling is critical for immune responses in microglia and macrophages that occur upon efferocytosis of dead/dying neurons. 
A

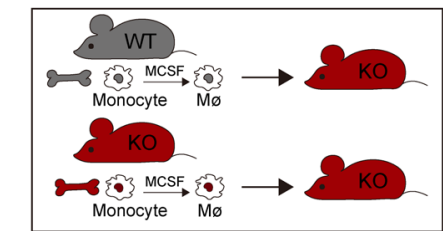

C

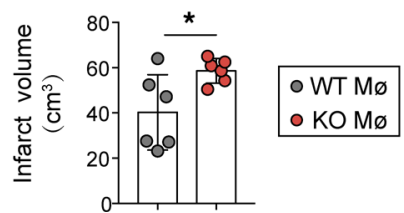

B

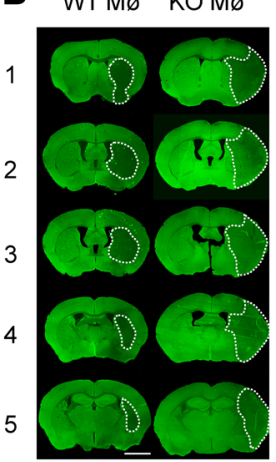

D

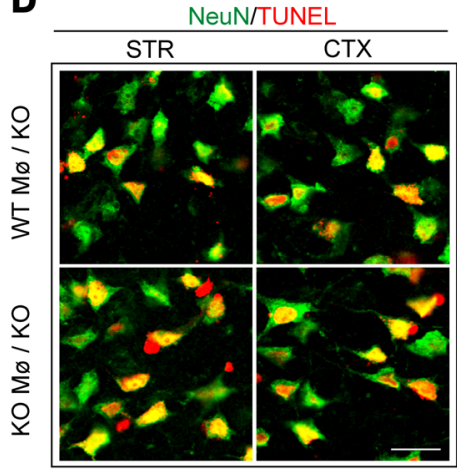

$\mathbf{E}$

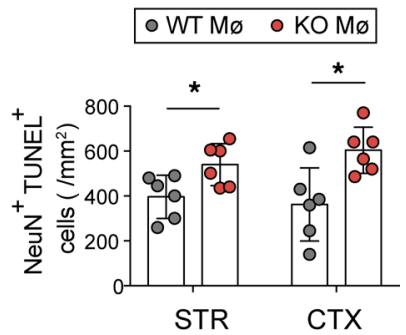

Figure 7. WT macrophage adoptive transfer improves stroke outcomes in STAT6-KO mice. (A) Two million macrophages (Mø) prepared from either WT mice or STAT6-KO mice were adoptively transferred (i.v. route) into the STAT6-KO mice 2 hours after 60 minutes of tMCAO. (B and C) Infarct volume at $3 \mathrm{~d}$ after tMCAO was quantified in MAP2-stained coronal sections. Dashed lines outline the infarct area. Scale bar: $1 \mathrm{~mm}$. $n=6 / \mathrm{group}$. (D and E) Neuronal death was quantified by TUNEL (red) and NeuN (green) colabeling in infarct areas $3 \mathrm{~d}$ after tMCAO. Scale bar: $20 \mu \mathrm{m}$. $n=6$ mice per group. ${ }^{*} P \leq 0.05$ vs. WT/WT, Student's $t$ test.

STAT6-mediated phagocytic activity in microglia and macrophages protects against ischemic neuronal death. Next, we explored whether STAT6 is essential for microglia-induced neuronal protection (Figure 9A). Primary neurons were subjected to OGD for 90 minutes, which killed $\sim 75 \%$ of neurons 24 hours later (Figure 9 , B-D). Immediately following OGD, primary microglia prepared from WT or STAT6-KO mouse pups were cocultured with the post-OGD neurons for 24 hours. Consistent with our previous report (17), WT microglia protected neurons against OGD-induced cell death, doubling the number of surviving neurons at 24 hours after OGD; however, STAT6-KO microglia failed to protect against OGD-induced neuron cell death (Figure 9, B and C). Furthermore, KO of STAT6 in microglia led to the loss of neuroprotection induced by the STAT6 inducer, IL-4 (Supplemental Figure 11). Similarly, STAT6-KO abolished macrophage-induced protection of neurons against ischemic injury (Figure 9D). Interestingly, inhibition of the phagocytic functions of WT microglia or macrophages by pretreating cells with $10 \mu \mathrm{M}$ cytochalasin D (an inhibitor of actin polymerization and phagocytosis) for 1 hour before coculture abolished their protective effects against OGD-induced neuronal death (Figure 9, B-D). Cytochalasin D pretreatment did not change the inability of STAT6-KO microglia to impact neuronal survival after OGD. Together, these data suggest that STAT6 signaling is essential for microglia/macrophage-afforded protection against ischemic neuronal injury and that this effect by microglia and macrophages may be, at least in part, attributable to their phagocytic function.

Arg1 is a downstream target of STAT6 signaling in microglia and macrophages. We noticed that Arg1, a molecule known to be important in macrophage-mediated protection against spinal cord injury (18), was expressed in both microglia and macrophages in the ischemic brain (Figure 6D), and STAT6-KO attenuated the poststroke induction of Arg1 in the brain (Figure 5F). We therefore investigated whether Arg1 is a downstream target of STAT6 and whether it is essential for the protective effect of STAT6 after stroke. Arg1 was expressed in $\mathrm{Iba}^{+}$microglia/macrophages in WT ischemic brain 3d after tMCAO (Figure 10A); however, Arg1 immunoreactivity was markedly decreased in $\mathrm{Iba}^{+}{ }^{+}$cells in postischemic STAT6-KO brains. Arg1 expression was minimal in resting microglia/macrophages in sham mice of either germ line (Supplemental Figure 5D). Using flow cytometry, we found that the MFI of Arg1 in CD11 $\mathrm{b}^{+} \mathrm{CD} 45^{+} \mathrm{pSTAT6}{ }^{+}$ microglia/macrophages was higher than that in the $\mathrm{CD} 11 \mathrm{~b}^{+} \mathrm{CD} 45^{+} \mathrm{pSTAT} 6^{-}$subset (Figure 10B), indicating that the activation of STAT6 is positively associated with Arg1 expression.

Compared with their WT counterparts, cultured STAT6-KO microglia and STAT6-KO macrophages showed no significant difference in the basal levels of Arg1 mRNA (Figure 10C) and protein (Figure 10D and Supplemental Figure 12) but did not show induction of Arg1 upon IL-4 stimulation. These data suggest that Arg1 may be a downstream target of STAT6 signaling in microglia and macrophages after stimulation.

Similar to STAT6-KO, the application of NOAH, an Arg1 inhibitor, inhibited the efferocytosis of dead/dying neurons by microglia and macrophages (Figure 11A). Arg1 inhibition also enhanced proinflammatory responses (i.e., increased IL- 6 and TNF- $\alpha$ production) in microglia exposed to dead/dying neurons but had a minimal effect on inflammatory responses in macrophages (Figure 11B). NOAH showed no effect on microglia and macrophage survival before or after phagocytosis (Figure 11C). 
A

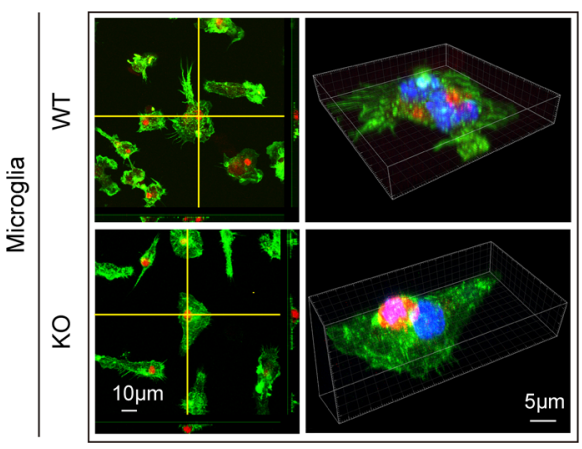

B

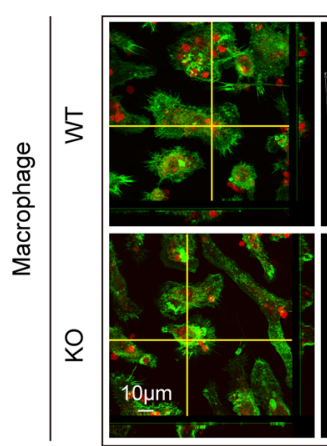

C

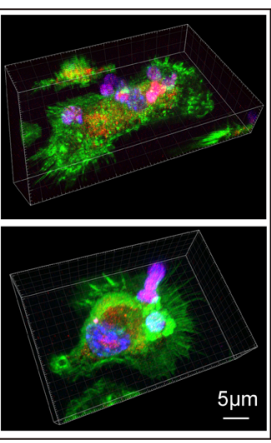

D
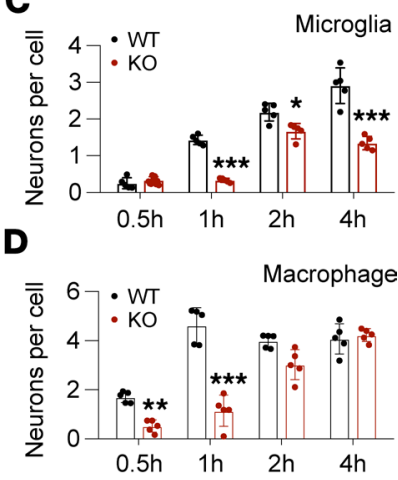
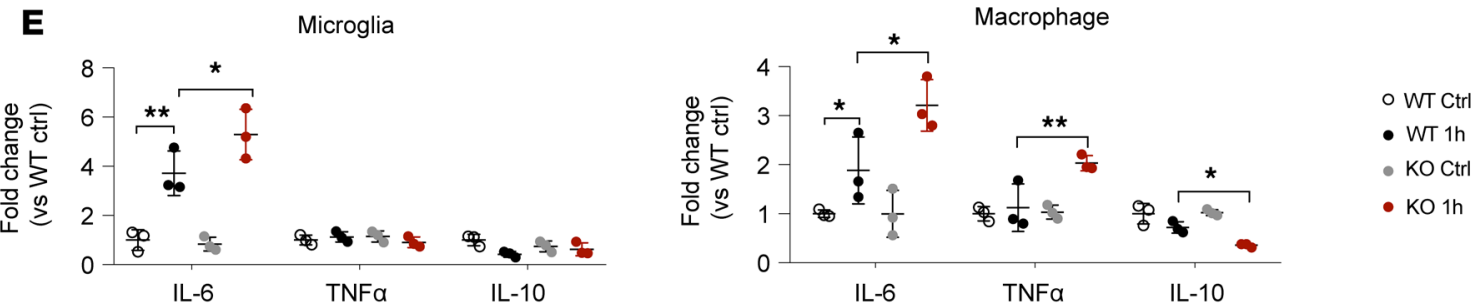

$\mathbf{F}$

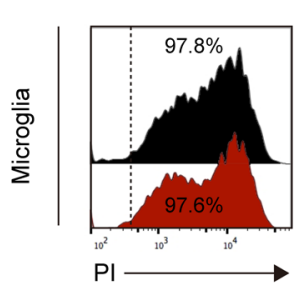

$\mathrm{PI}^{+} \mathrm{CD} 11 \mathrm{~b}^{+}$gated
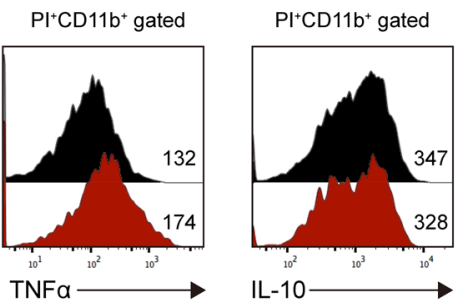

- WT $\cdot$ KO
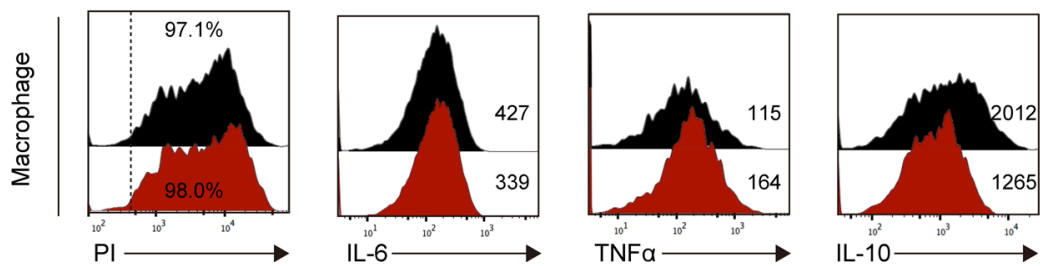

G

Microglia

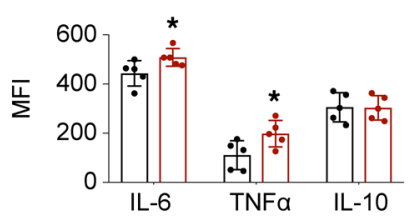

H

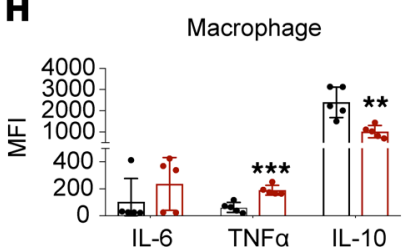

Figure 8. STAT6 signaling facilitates efferocytosis and antiinflammatory responses in both microglia and macrophages. (A-D) PI-labeled dead/dying neurons were added to WT or STAT6-KO microglia or macrophages. (A and B) Representative images showing phagocytosis of $\mathrm{Pl}^{+}$dead/dying neurons (red) in phalloidin-labeled microglia (green, A) or macrophages (green, B). Blue shows DAPI staining of nuclei. (C and D) Quantification of engulfed $\mathrm{PI}^{+}$neurons in microglia (C) and macrophages (D) at indicated time points after coincubation. Data represent 5 independent experiments in duplicate. ${ }^{*} P \leq 0.05,{ }^{* *} P \leq$ $0.01,{ }^{* * *} P \leq 0.001 \mathrm{vs}$. WT at corresponding time points, Student's $t$ test. (E) RT-qPCR analysis of mRNA expression of proinflammatory (IL-6 and TNF- $\alpha$ ) and antiinflammatory (IL-10) factors in microglia and macrophages at 1 hour after efferocytosis of $\mathrm{Pl}^{+}$dead/dying neurons. Data represent 3 independent experiments in duplicate. ${ }^{*} P \leq 0.05,{ }^{*} P \leq 0.01$, 1-way ANOVA. (F-H) Flow cytometry analysis of protein expression of proinflammatory (IL-6 and TNF- $\alpha$ ) and antiinflammatory (IL-10) factors in microglia and macrophages 6 hours after incubation with $\mathrm{Pl}^{+}$dead/dying neurons. Data represent 5 independent experiments in duplicate. ${ }^{*} P \leq 0.05,{ }^{* *} P \leq 0.01,{ }^{* * *} P \leq 0.001$ vs. WT, Student's $t$ test.

To confirm that Arg1 is a downstream molecule of STAT6 that critically regulates microglia/macrophage phagocytosis, we overexpressed Arg1 in cultured microglia and macrophages from STAT6-KO mice. In agreement with results in Figure 8, C and D, loss of STAT6 expression markedly impaired the efferocytosis of dead/dying neurons by microglia and macrophages, respectively (Figure 11, D and E). Lentivirus-mediated overexpression of Arg1 in STAT6-KO microglia and macrophages (Supplemental Figure 13) completely restored their efferocytic capacities (Figure 11, D and E). Arg1 overexpression did not significantly impact cell viability of microglia or macrophages (Figure 11F).

STAT6 deficiency impairs long-term recovery after stroke. Finally, we evaluated the role of STAT6 in long-term brain injury and functional deficits after ischemic stroke. As shown in Figure 12A, STAT6$\mathrm{KO}$ mice exhibited significantly enlarged brain tissue loss compared with WT mice $35 \mathrm{~d}$ after tMCAO. 
A

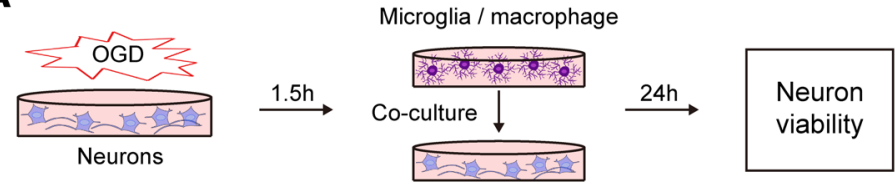

B No OGD

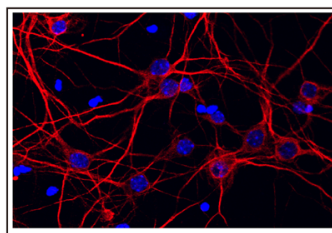

OGD+Veh

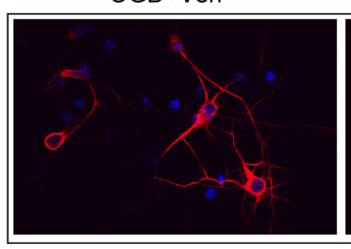

OGD+WT

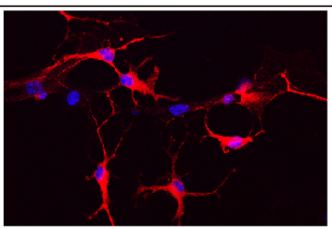

OGD+KO

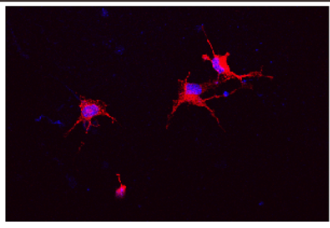

OGD+WT-Cytocalasin D

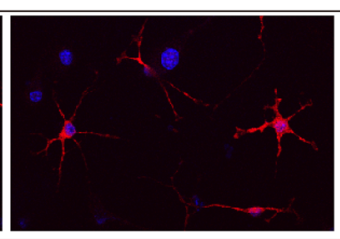

OGD+KO-Cytocalasin D

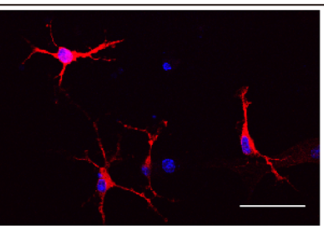

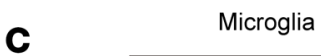

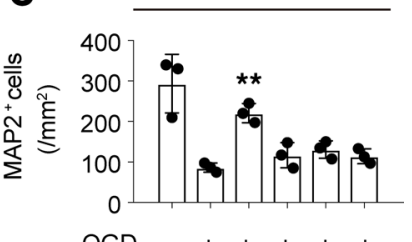

OGD - + + + + +

Microglia - - WTWTKO KO

Cytochalasin D

pre-treatment $/$ / +-+

Dacrophage

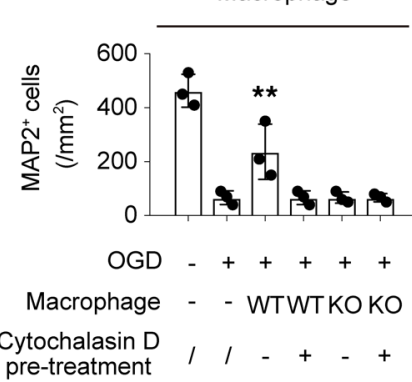

Figure 9. STAT6 deficiency negates microglia/macrophage-induced protection of ischemic neurons. (A) Experimental design. Primary neurons were subjected to 90-minute OCD and then cocultured with WT or STAT6-KO microglia/macrophages. Neuronal survival was measured 24 hours later by MAP2 staining. (B) Representative images of MAP2-stained neurons after coculturing with WT or STAT6-KO microglia. Scale bar: $50 \mu \mathrm{m}$. (C and D) The number of MAP2 ${ }^{+}(\mathrm{red})$ neurons was quantified 24 hours after coculturing with WT or STAT6-KO microglia (C) or macrophages (D). In some groups, WT microglia or macrophages were treated with cytochalasin $\mathrm{D}\left(10 \mu \mathrm{M}, 1\right.$ hour) to inhibit phagocytosis. Data represent 3 independent experiments in duplicate. ${ }^{* *} P \leq 0.01 \mathrm{vs}$. OGD alone (2nd bar), 1-way ANOVA.

The sensorimotor deficits after stroke were exacerbated in STAT6-KO mice, as shown by increased neurological deficit scores, reduced time in the rotarod test, and increased number of contralateral forelimb foot-faults (Figure 12, B-D). STAT6-KO mice also showed impaired spatial learning ability compared with WT mice after stroke (Figure 12E), as revealed by their increased time to find the hidden platform in the Morris water maze test. Spatial memory capacity in STAT6-KO stroke mice, as measured by the time spent in the target quadrant (platform area) when the platform was removed, was similar to WT stroke mice (Figure 12E). No differences in sensorimotor or cognitive functions were observed between WT and STAT6-KO sham control mice (Supplemental Figure 5, B and C).

\section{Discussion}

The present study identifies STAT6 as a key transcription factor that mediates the effective efferocytosis and antiinflammatory responses of microglia and macrophages early after stroke. STAT6 deficiency impairs the clearance of dead/dying neurons, exacerbates local inflammation, enlarges brain tissue loss, and worsens long-term functional deficits. The activation of STAT6 in the ischemic brain has been previously reported in experimental animals at 3-12 hours after tMCAO and in brain slices from stroke patients $(14,19)$. However, the specific cell type in which this activation occurs has not been previously identified. In our animal study, STAT6 activation was detected in microglia/macrophages, but not in other types of CNS cells, early after stroke. The BM chimeras with STAT6-KO in the CNS or periphery further confirmed the essential role of STAT6 activation in microglia and macrophages in improving stroke outcomes. In stroke patients, we also observed increased pSTAT6 signal in microglia/macrophages in the ischemic brains. Interestingly, pSTAT6 signal was detected in Iba1 ${ }^{-}$cells in both stroke patients and nonstroke aged brain. This is consistent with a previous report by Jung et al. that documented STAT6 expression in the peri-infarct region of brains from stroke patients (14). Jung et al.'s study - while it did not use cellular markers to specify cell type in human samples - reported STAT6 activation in oxidatively stressed neurons in culture, with elevated STAT6 activity leading to neuronal death. However, the lack of pSTAT6 in neurons in our in vivo stroke model suggests that the effect of STAT6 signaling in neurons is minimal, at least at early time points after stroke. It is also important to consider the expression of STAT6 in peripheral immune cells other than macrophages. In particular, STAT6 is expressed in T lymphocytes and is essential for T cell differentiation 
A

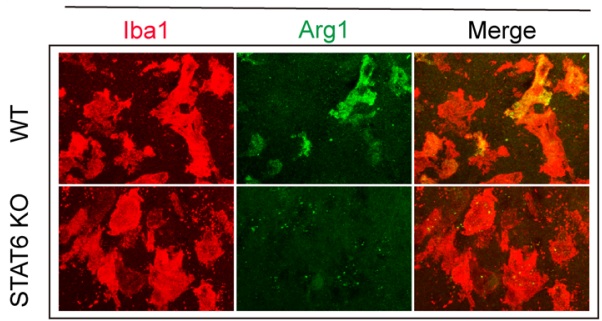

C

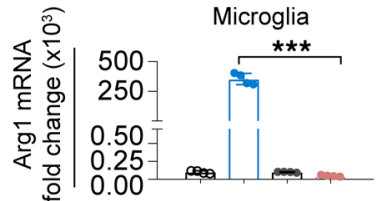

OWT+Veh $\cdot \mathrm{WT}+\mathrm{IL}-4 \cdot \mathrm{KO}+\mathrm{Veh} \cdot \mathrm{KO}+\mathrm{IL}-4$

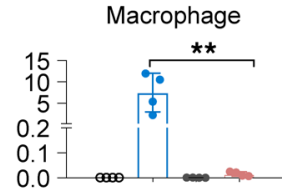

CTX

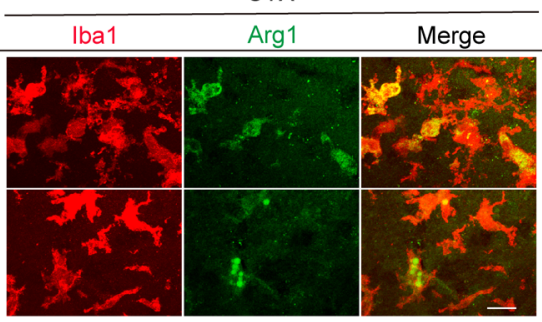

D

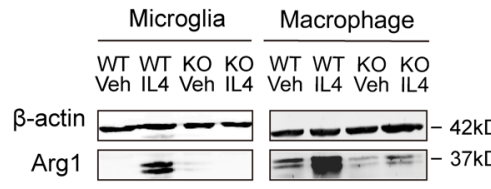

B
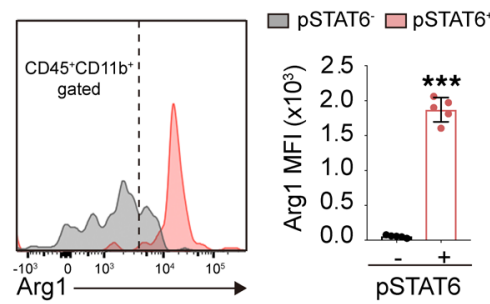

$\circ \mathrm{WT}+\mathrm{Veh} \cdot \mathrm{WT}+\mathrm{IL}-4 \cdot \mathrm{KO}+\mathrm{Veh} \cdot \mathrm{KO}+\mathrm{IL}-4$

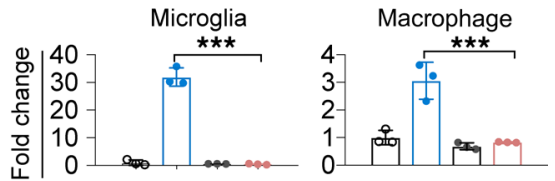

Figure 10. Arginase 1 is a downstream target of STAT6 signaling in microglia and macrophages. (A) Immunostaining of Arginase 1 (Arg1, green) in Iba1 ${ }^{+}$microg$\mathrm{lia/macrophages} \mathrm{(red)} \mathrm{in} \mathrm{the} \mathrm{ischemic} \mathrm{striatum} \mathrm{(STR)} \mathrm{and} \mathrm{cortex} \mathrm{(CTX)} \mathrm{3d} \mathrm{after} \mathrm{cerebral} \mathrm{ischemia.} \mathrm{Scale} \mathrm{bar:} 20 \mu \mathrm{m}$. (B) The expression of Arg1 in pSTAT6 ${ }^{+}$(pink) and pSTAT6- (gray) microglia/macrophage (CD45+CD11 ${ }^{+}$gated) was measured 3d after tMCAO in ipsilateral hemisphere of WT mice by flow cytometry. Mean fluorescent intensity (MFI) of Arg1 was quantified. $n=5$ mice. ${ }^{* * *} P \leq 0.001$ vs. pSTAT6- population, Student's $t$ test. (C) RT-qPCR analysis of Arg1 mRNA expression in WT and STAT6-KO microglia or macrophages without stimulation and after activating STAT6 signaling with IL-4 (20 ng/mL for 48 hours). Data were collected from 4 independent experiments. ${ }^{* *} P \leq 0.01,{ }^{* *} P \leq 0.001,1$-way ANOVA. (D) Western blot analysis of Arg1 protein expression in microglia or macrophages without stimulation and after activating STAT6 signaling with IL-4. Data were collected from 3 independent experiments. ${ }^{* * *} P \leq 0.001,1$-way ANOVA.

toward an antiinflammatory Th2 subtype (20). The worsened outcome observed after stroke in chimeric mice with peripheral STAT6-KO confirmed the involvement of peripheral STAT6 signaling on stroke outcomes but cannot distinguish between the contribution of STAT6 in macrophages vs. T lymphocytes. However, we showed that adoptive transfer of WT macrophages reinstated the capacity to clear dead neurons by phagocytes $3 \mathrm{~d}$ after stroke and reduced brain infarction in STAT6-KO mice; this observation strongly supports a major role of STAT6 in microglia/macrophage efferocytosis and brain lesion after stroke. Our in vitro data further confirm the importance of macrophage-derived STAT6 to ischemic neurons.

This study discovered that STAT6 activation enhances the efferocytic activities and inhibits inflammatory responses of microglia and macrophages. Although microglia and macrophages displayed similar responses in the presence of dead/dying neurons, differences in their STAT6 expression and functions were noted. First, the data generated from WT mice (Figure 1) and WT/CX3CR1-GFP chimeric mice (Figure 6) demonstrated a high expression level of pSTAT6 on macrophage in the ischemic brain, suggesting a predominant role of STAT6 in macrophages. This may at least partially explain why STAT6-KO caused more widespread disturbance of cytokine production (e.g., IL-6, TNF- $\alpha$, and IL-10) in macrophages, whereas fewer cytokines (e.g., IL-6) were influenced in microglia in cultures. Second, macrophages responded much faster than microglia when encountering dead/dying neurons in vitro, as manifested by earlier onset and plateau of efferocytosis. Interestingly, STAT6 expression in macrophages only influenced efferocytosis at early stages (0.5-1 hours) after being cocultured with dead/dying neurons. In contrast, STAT6 expression in microglia impacted efferocytosis at longer time periods after being cocultured with dead/dying neurons. Finally, we observed similar effects of STAT6 on phagocytosis of large particles (dead/dying neurons) and small fluorescently labeled beads $(1 \mu \mathrm{m})$ in microglia, whereas a previous study in macrophages showed that STAT6 activation enhanced phagocytosis of large beads and limited the engulfment of small beads (21). These differences suggest that STAT6 expression in microglia and macrophages might perform their phagocytic roles in different manners. STAT6 might be critical in maintaining the efferocytic capacity of microglia but may be essential for the quick initiation of efferocytosis in infiltrating macrophages in the ischemic brain. It is also worth noting that, although our study focused on STAT6-mediated clearance of dead/dying neurons, the effects of STAT6 on efferocytosis were not specific to dead/dying neurons. As mentioned earlier, STAT6-KO microglia under IL-4 stimulation exhibited impaired phagocytosis of latex beads, suggesting a nonspecific effect of STAT6 on phagocytosis of microglia.

Phagocytosis is not always beneficial in injured brains. Improper phagocytosis of stressed neurons by microglia may execute the death of these otherwise viable cells (22). This so-called phagoptosis is more active 
A

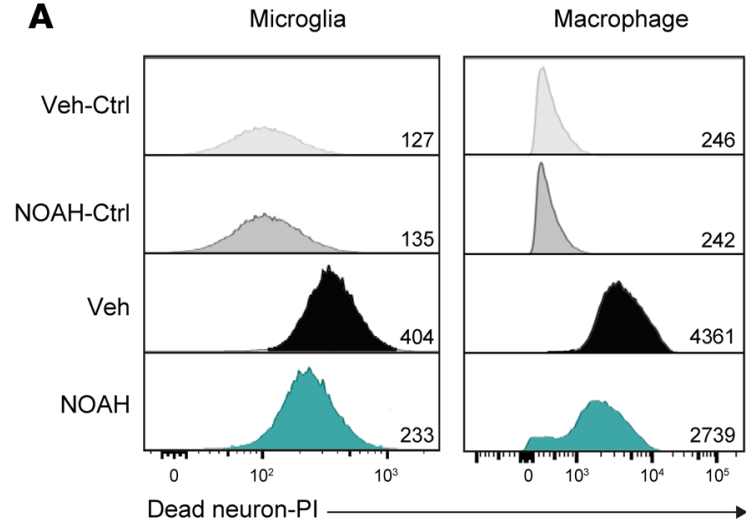

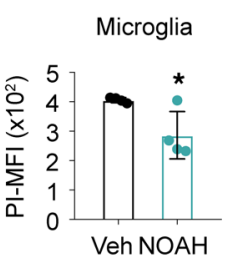

Macrophage

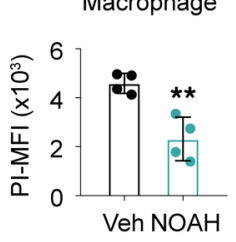

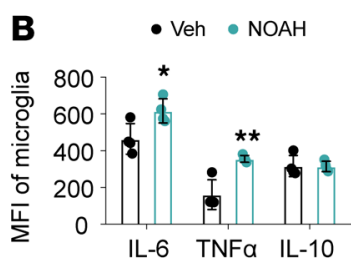
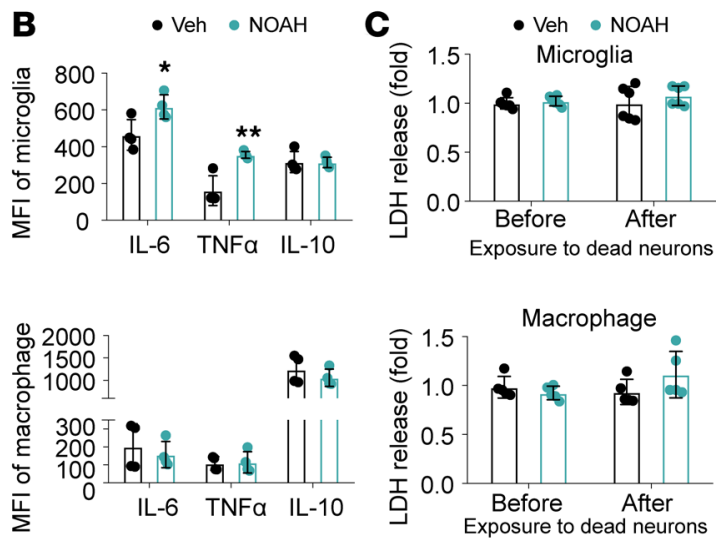

D

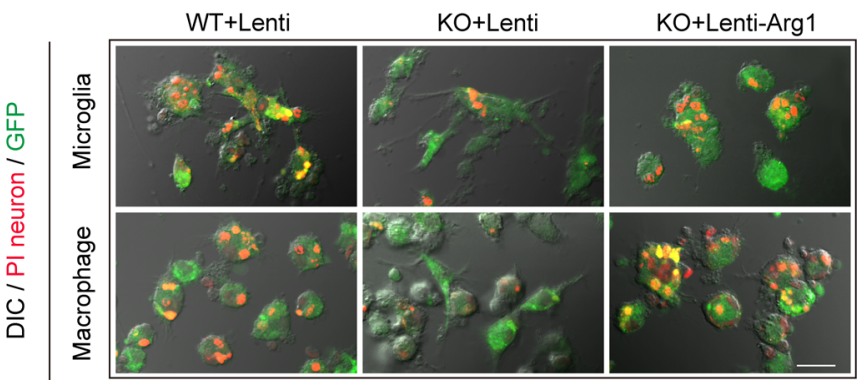

$\mathbf{E}$

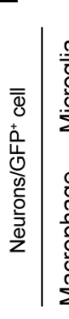

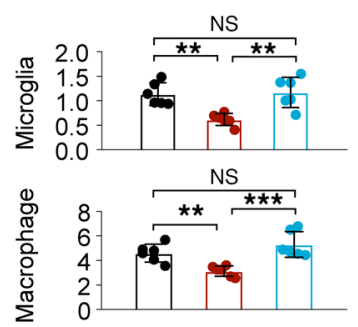

- WT+Lenti • KO+Lenti • KO+Lenti-Arg1

$\mathbf{F}$

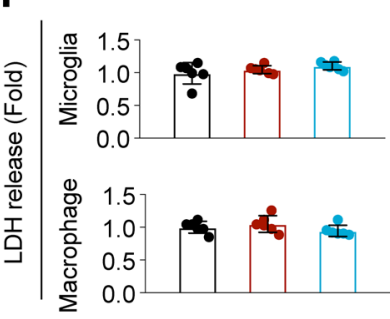

Figure 11. Arg1 is essential for efferocytic activity of primary cultured microglia and macrophages. NOAH, an arginase inhibitor, or vehicle control was applied to microglia and macrophages for 1 hour before exposure to $\mathrm{Pl}^{+}$dead/dying neurons. (A) Phagocytosis of $\mathrm{Pl}^{+}$dead/dying neurons by microglia or macrophages was quantified as MFI of PI in microglia or macrophages using flow cytometry. Data were collected from 4 independent experiments. ${ }^{*} P \leq$ $0.05,{ }^{* *} P \leq 0.01$ NOAH vs. vehicle, Student's $t$ test. (B) Protein expression of proinflammatory (IL-6 and TNF- $\alpha$ ) and antiinflammatory (IL-10) factors in microglia and macrophages was quantified by flow cytometry at 6 hours after efferocytosis. Data were collected from 4 independent experiments. ${ }^{*} P \leq$ $0.05,{ }^{* *} P \leq 0.01 \mathrm{NOAH}$ vs. vehicle, Student's $t$ test. (C) Cell death was quantified by the LDH assay. Data were collected from 6 independent experiments for microglia and 5 independent experiments for macrophage. (D-F) STAT6-KO microglia and macrophages were infected with lentiviral vectors carrying Arg1 cDNA and GFP (Lenti-Arg1) or control lentivirus carrying GFP only (Lenti) for 2d and incubated with PI-labeled post-OCD neurons. Phagocytosis of PI dead/dying neurons by Lenti-GFP-transfected WT (WT+Lenti), Lenti-GFP-transfected STAT6-KO (KO+Lenti), or Lenti-Arg1-GFP-transfected STAT6-KO (KO+Lenti-Arg1) microglia and macrophages was quantified 4 hours (microglia) and 1.5 hours (macrophage) after coculture. (D) Representative images of microglia/macrophage (green) phagocytosis of dead/dying neurons (red) overlaid on DIC images. Scale bar: $20 \mu \mathrm{m}$. (E) Quantification of the number of engulfed $\mathrm{Pl}^{+}$neurons observed in $\mathrm{GFP}^{+}$microglia or GFP+ macrophages. (F) Quantification of $\mathrm{LDH}$ release in conditioned medium collected from microglia or macrophages after virus transfection. Data represent 6 independent experiments in duplicate. ${ }^{* *} P \leq 0.01,{ }^{* * *} P \leq 0.001,1$-way ANOVA.

in inflammatory environments, as inflammation causes the exposure of "eat-me" signals in stressed-but-viable neurons. One concern related to this phenomenon is whether STAT6-enhanced engulfment of damaged neurons is an over-commitment and might actually be detrimental to stroke outcome. This possibility was disputed by data that showed that the addition of WT, but not STAT6-KO, microglia/macrophages indeed enhanced neuronal survival after OGD. In contrast, in the presence of cytochalasin D, microglia/macrophages failed to provide any neuronal protection. These data provide compelling evidence that phagoptosis is not active in the OGD neuron-microglia/macrophage coculture system and that STAT6-enhanced efferocytosis is not related to increased neuronal death. The in vivo data further confirmed this assertion, as more than $80 \%$ of engulfed neurons were TUNEL ${ }^{+}$dead/dying neurons, and STAT6 deficiency resulted in an accumulation of dying neurons in peri-infarct areas in STAT6-KO stroke mice. Only a small number of cells were $\mathrm{Iba}^{+}{ }^{+} \mathrm{NeuN}^{+} \mathrm{TUNEL}^{-}$, which may reflect surveillant contact between microglia and neurons.

An outstanding question is what mechanisms underlie STAT6-mediated efferocytosis and antiinflammatory responses in microglia and macrophages. Previous studies have documented that activation of STAT6 augmented PPAR $\gamma$ activity and facilitated the expression of PPAR $\gamma$-regulated genes (23). PPAR $\gamma$ is known to be critical for macrophage efferocytosis and phagocytosis by upregulating the expression of phagocytic receptors, including CD36 (24-26), and other recognition receptors (27). STAT6-dependent expression of E-cadherin is also known to be involved in phagocytosis of large particles by regulating cell-to-cell interactions and the fusion among neighboring macrophages (21). Effective efferocytosis sequesters dead or 

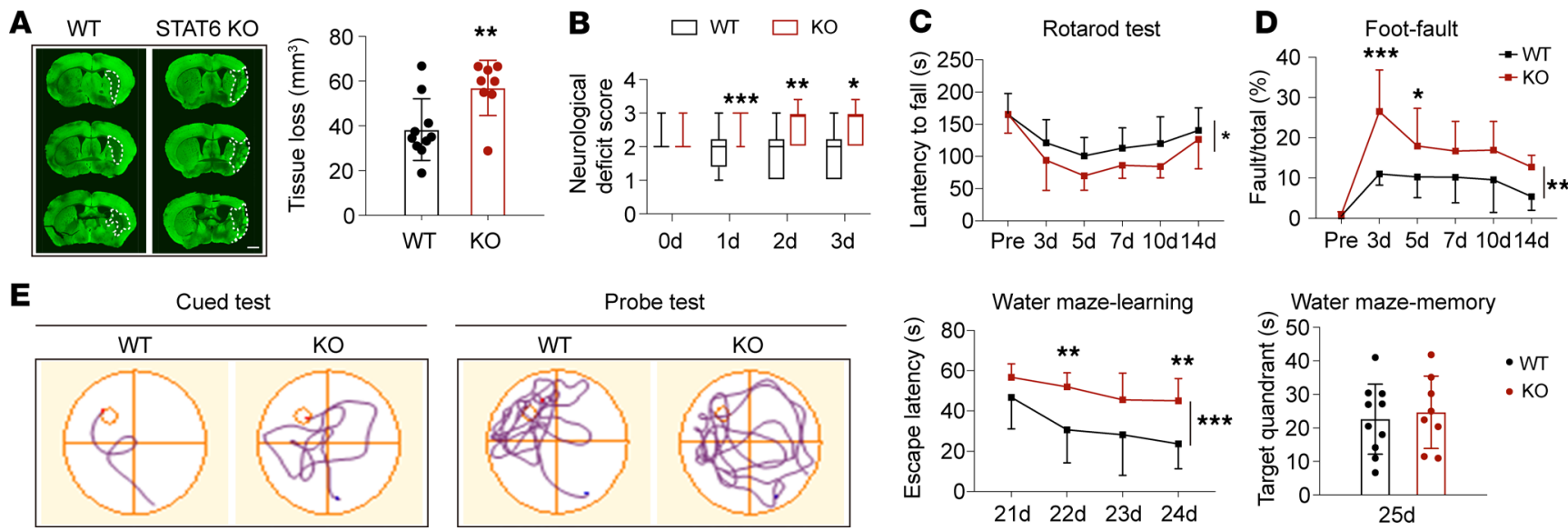

Figure 12. STAT6 deficiency impairs long-term recovery after tMCAO. WT and STAT6-KO mice were subjected to 60 minutes of tMCAO. (A) Brain tissue loss 35d after tMCAO was quantified on MAP2-stained (green) coronal sections. $n=8-10$ mice per group. Scale bar: 1 mm. (B) Neurological deficit score was assessed during the first 3d after tMCAO. (C and D) Sensorimotor deficits were evaluated with rotarod (C) and foot-fault (D) tests 3d-14d after tMCAO. (E) Cognitive function was assessed with Morris water maze at 21d-25d after tMCAO. Latency to find the hidden platform in the cued test (spatial learning) and time spent in the target quadrant in the probe test (memory) were measured. $n=8-10$ mice per group. ${ }^{*} P \leq 0.05$, ${ }^{* *} P \leq 0.01,{ }^{* *} P \leq 0.001$ vs. WT, Student's $t$ test $(\mathbf{A})$, Mann-Whitney $U$ test (B), or 2-way ANOVA repeated measurement (C-E).

dying cells to reduce the release of damage-associated molecular patterns and ameliorate inflammation. In addition, efferocytosis engages several metabolic-sensing molecules such as LXR $\alpha, \operatorname{LXR} \beta, \operatorname{PPAR} \gamma, \operatorname{PPAR} \delta$, $\mathrm{RXR} \alpha$, and the CD73-adenosine receptor A2a axis to control inflammatory responses in phagocytes and promotes inflammation resolution $(28,29)$. Previous studies suggested that Arg1 might be a downstream target regulated by STAT6 $(30,31)$. In this study, we also found that Arg1 is a STAT6-regulated molecule. Arg1 is an enzyme that catalyzes the conversion of arginine to ornithine and urea. As it competes with inducible nitric oxide synthase (iNOS) for the substrate of arginine, it effectively downregulates the production of nitric oxide (32) and, thus, eases the burden of intracellular oxidative stress during various cell activities. Arg1 mainly expresses in neurons in a normal brain. The expression of Arg1 increases in microglia/macrophages and astrocytes early after CNS injuries $(33,34)$. Arg1 has been regarded as a marker for beneficial microglia/macrophages and possesses antiinflammatory and tissue repair properties under various pathological conditions (17). Whereas a recent study using models of spinal cord injury and experimental autoimmune encephalomyelitis showed Arg1 expression exclusively in infiltrated leukocytes (35), our results using BM chimeric mice clearly revealed elevated Arg1 expression in both macrophages and microglia, suggesting the importance of Arg1 in both cell populations in a stroke model. The Arg $1^{+}$phenotype of microglia has been shown to protect against neuronal death and augment neurite growth after injury $(17,18)$. Arg $1^{+}$microglia are also involved in reducing $\mathrm{A} \beta$ plaque and inhibiting local inflammation in $\operatorname{AD}$ models $(29,36)$. Our experiment discovered the importance of Arg1 in promoting efferocytosis and inhibiting inflammatory responses after efferocytosis in microglia. Interestingly, it appears that Arg1 is important for inflammatory responses after dead/dying neuron phagocytosis in microglia but not in macrophages. This observation indicates that, like STAT6, Arg1 also performs important roles in different manners in microglia and macrophages. With the ability to enhance phagocytosis, limit inflammation in microglia/macrophages after phagocytosis, and promote brain repair (18), Arg1 may represent a novel target for stroke treatment.

In summary, the current study identifies STAT6 as a key molecule that mediates efferocytic activity of microglia/macrophages and promotes inflammation resolution in the ischemic brain. We also elucidated a mechanism whereby Arg1 contributes to STAT6-mediated protective responses in microglia/macrophages. The STAT6/Arg1 signaling may be a viable therapeutic target to regulate microglia/macrophage responses in the ischemic brain, mitigate brain injury, and promote long-term favorable outcomes.

\section{Methods}

Human material. Paraffin sections from 2 stroke patients and a control individual with no history of stroke were obtained from the University of Pittsburgh Neuropathology Brain Bank. Stroke patient 1 was an 83-year-old male who suffered from an acute ischemic stroke in MCA territory (section obtained from parietal cortex). 
Stroke patient 2 was an 88-year-old female with an acute/early subacute infarct in the superior temporal cortex. Tissue sections were used for immunological staining as approved by the Committee for Oversight of Research and Clinical Training Involving Decedents (CORID; no. 914).

Animals. C57BL/6J WT, STAT6-KO (STAT6/-, KO), and CX3CR1-GFP mice were purchased form the Jackson Laboratory. Mice were housed in a temperature- and humidity-controlled and specific pathogen-free animal facility with a 12-hour light-dark cycle at the University of Pittsburgh. Food and water were available ad libitum. Mice were randomly assigned to experimental groups using a lottery drawing box. All efforts were made to minimize animal suffering.

Murine models of transient cerebral ischemia. Transient cerebral ischemia was induced by intraluminal occlusion of the left MCA for 60 minutes as described previously (37). Female mice (WT or STAT6-KO, 6-8 weeks old) were OVX 2 weeks before transient cerebral ischemia. Eight- to 10-week-old male (25-30 g) or OVX female mice (20-25 g) were subjected to tMCAO. Rectal temperature was controlled at $37.0^{\circ} \mathrm{C}$ $\pm 0.5^{\circ} \mathrm{C}$ during surgery using a temperature-regulated heating pad. $\mathrm{rCBF}$ was measured in all stroke animals using the 2-D laser speckle imaging system (PeriCam PSI System, Perimed) and/or laser Doppler flowmetry. Animals that died or did not show at least 70\% rCBF reduction of preischemia levels using laser Doppler flowmetry were excluded from further experimentation. Sham-operated animals underwent the same anesthesia and exposure of arteries without MCA occlusion. Investigators who were blinded to animal genotype and experimental grouping performed surgery of tMCAO.

A total of 75 WT (65 male and 10 female) and 80 STAT6-KO mice (68 male and 12 female) were subjected to tMCAO. The mortality rate was $6.2 \%$ (4 of 65) in WT male, $20.0 \%$ (2 of 10) in WT female, $10.3 \%$ (7 of 68) in STAT6-KO male, and 8.3\% (1 of 12) in STAT6-KO female. There was no significant difference in mortalities between WT and STAT6-KO groups after stroke. A total of 36 chimera mice (12 WT/WT, $12 \mathrm{WT} / \mathrm{KO}$, and $12 \mathrm{KO} / \mathrm{WT}$ ) were subjected to tMCAO. The mortality rate was $16.6 \%$ (2 of 12 ) in WT/ WT mice, $25 \%$ (3 of 12) in WT/KO, and 25.0\% (3 of 12) in KO/WT. There was no significant difference in mortalities among different chimera groups after stroke. A total of $6 \mathrm{WT}$ (male) and 6 STAT6-KO (male) mice were subjected to sham operation. No animals died from the sham procedure.

Irradiation and BM transplantation. To construct BM chimeric mice, 5-week-old recipient male C57BL/6J WT and STAT6-KO mice were anesthetized and exposed to lethal $\gamma$ irradiation ( $950 \mathrm{rad}, 10 \mathrm{~min}, 1$ dose) with their heads shielded (38). BM cells obtained from 8- to 10-week-old male C57BL/6J, STAT6-KO, or CX3CR1-GFP donors were i.v. transferred to recipients $\left(1 \times 10^{7}\right.$ cells per recipient $) 6$ hours after irradiation. Chimeric mice were allowed to recover for 6 weeks before brain ischemia induction. Based on our experience, the degree of chimerism in recipient mice is about $90 \%$, as tested by flow cytometry in blood macrophages.

Cortical cerebral blood flow measurements. rCBF was measured in tMCAO animals using the 2-D laser speckle imaging system (PeriCam PSI System; Perimed) as previously reported (37). WT and STAT6-KO mice were subjected to repeated measurements of $\mathrm{CBF} 15$ minutes before $\mathrm{tMCAO}$, during tMCAO, and 15 minutes after the onset of reperfusion. $\mathrm{CBF}$ changes were expressed as a percentage of pre-tMCAO baseline.

Measurement of infarct volume. Six equally spaced coronal brain sections encompassing the MCA territory were stained with anti-microtubule associated protein 2 (MAP2, a neuron-specific marker) antibody (MAB3418, 1:300; MilliporeSigma) to visualize lived brain tissue. Infarct volume was analyzed by a blinded observer using NIH ImageJ software on MAP2-stained sections. The infarct volume with corrections for edema was calculated as the volume of the contralateral hemisphere minus the noninfarcted volume of the ipsilateral hemisphere.

IHC and image analysis. Coronal brain sections $(25 \mu \mathrm{m})$ were cut on a freezing microtome and subjected to immunofluorescence staining. The following primary antibodies were used: rabbit polyclonal anti-pSTAT6 (phospho Y641) (Ab28829, Abcam, 1:100, used for both mouse and human samples); goat polyclonal antiIba1 (Ab5076, Abcam, 1:500, used for both mouse and human samples); rabbit polyclonal anti-Iba1 (019. 19741, Wako, 1:500); mouse anti-NeuN (MAB377, MilliporeSigma,1:500); rabbit anti-NeuN (ABN78, MilliporeSigma,1:500); rat anti-CD16/CD32 (553142, BD Biosciences, 1:200); goat anti-CD206 (AF2535, R\&D Systems,1:500); and goat polyclonal anti-Arg1 (sc-18351, Santa Cruz Biotechnology Inc., 1:200). The following secondary antibodies (all from Jackson ImmunoResearch Laboratories) were applied: anti-goat secondary antibody conjugated with Cy3 (705165033), anti-rabbit secondary antibody conjugated with Сy3 (711165152), anti-rabbit secondary antibody conjugated with Alexa Fluor 488 (711545152), anti-rabbit secondary antibody conjugated with Alexa Fluor 405 (711475152), anti-rat secondary antibody conjugated with Alexa Fluor 488 (712545150), and anti-mouse secondary antibody conjugated with Alexa Fluor 488 (715545150). Sections were mounted with DAPI Fluoromount-G or Fluoromount-G (Southern Biotech). For immunostaining with 
the use of mouse-origin primary antibodies against mouse tissues, M.O.M. kit (BMK-2202, Vector Laboratories) was applied before primary antibodies to block nonspecific signals, according to the manufacturer's instructions. For neuronal apoptosis analysis, TUNEL was processed after NeuN labeling according to instructions from the manufacturer (In Situ Cell Death Detection Fluorescein, 11684795910, Roche Diagnostics). Fluorescence images were captured using confocal microscopy (FV1000, Olympus Tokyo).

Images of 2 randomly selected microscopic fields in the cortex and 2 in the striatum in the peri-infarct areas of each section were subjected for analysis. The border of the infarct was determined by the loss of $\mathrm{NeuN}$ staining and the accumulation of $\mathrm{Iba}^{+}$microglia/macrophages. The stroke peri-infarct area was defined as the region that covers a radial distance of approximately $300-400 \mu \mathrm{m}$ from the border of infarct. Two to 3 sections covering the infarct area were assessed for each mouse brain. For microglia/macrophage quantification, the recorded images were loaded onto ImageJ (NIH) and were manually quantified by 2 observers blinded to grouping. Positively stained cells were electronically labeled with the software to avoid reduplicated counting. Data were expressed as mean number of cells per square millimeter.

For TUNEL and NeuN double-staining, the clearance rate of dead/dying neurons in each genotype (WT or STAT6-KO) was calculated as ([the mean number of $\mathrm{NeuN}^{+} \mathrm{TUNEL}^{+}$cells on day 3 - the number of $\mathrm{NeuN}^{+} \mathrm{TUNEL}^{+}$cells on day 7 in each mouse]/ [mean number of $\mathrm{NeuN}^{+} \mathrm{TUNEL}^{+}$cells on day 3] $\times 100 \%$ ). For analysis of dead/dying neuron clearance, brain slices were triple-stained with Iba1/NeuN/TUNEL. Images were processed with Imaris (Bitplane) for cell-based counting of automatically recognized cells. Briefly, the acquired data set was imported into Imaris. The spot detection algorithm was applied to assign a spot for each fluorescent intensity of a single nucleus. The settings for the spot detection algorithm were: [algorithm] enable region of interest $=$ false; enrich region growing $=$ false; enable tracking $=$ false; [source channel] source channel index $=1$; estimated diameter $=10.0 \mu \mathrm{m}$; background subtraction $=$ True; [Classify Spots] "quality" above 7.5. Cells with diameter $\geq 10.0 \mu \mathrm{m}$ were recognized as 1 cell. Positively stained cells were counted from 2 microscopic fields in the cortex and 2 in the striatum of each section. Three-dimensional images were reconstructed with Imaris from z-stack scanned OIB documents obtained from confocal microscopy. To view inside the cell, the surface was cut using the clipping plan. The surface effects were applied in the 3-D rendering to show the structures in the planes.

Flow cytometry. Flow cytometric analysis was performed using a FACS flow cytometer (BD Biosciences) as describe previously (39). To determine the expression of inflammatory factors in microglia or macrophage by FACS after efferocytosis, primary microglia or macrophage cultures were first subjected to efferocytosis assays (described below) for 6 hours and then fixed and permeabilized with intracellular staining Kit (Thermo Fisher Scientific). Cells were then stained with antibodies against inflammatory factors (IL- 6 , TNF- $\alpha$ and IL-10) at room temperature for 30 minutes. Antibodies used in flow cytometric staining were listed as below: rabbit polyclonal anti-NeuN (sc-20172, Santa Cruz Biotechnology Inc., 1:100), anti-rabbit IgG PerCP-Cy5.5 (sc-45101, Santa Cruz Biotechnology Inc., 1:100); anti-STAT6 (pY641) Alexa Fluor 488 (558243, BD Biosciences, 1:100), anti-O4-APC (130-109-153, Miltenyi Biotec, 1:10), anti-GLAST-PE (130-098-804, Miltenyi Biotec, 1:10), anti-CD45-VioBlue (130-102-775, Miltenyi Biotec, 1:10), anti-CD11b-Vio770 (130109-356, Miltenyi Biotec, 1:10), anti-CD206-Alexa Fluor 647 (565250, BD Biosciences, 1:150), anti-CD16BUV737 (565272, BD Biosciences, 1:400), anti-arginase 1-PE (IC5868P, R\&D System, 1:150), anti-IL6-PE (12-7061-82, Thermo Fisher Scientific, 1:100), anti-TNF- $\alpha-A P C$ (17-7321-82, Thermo Fisher Scientific, 1:100), and anti-IL10-PerCP Cy5.5 (45-7101-82, Thermo Fisher Scientific, 1:100). Appropriate isotype controls were stained following the manufacturer's instruction (01-1111-41, Thermo Fisher Scientific). Fluorochrome compensation was performed with single stained OneComp eBeads (Thermo Fisher Scientific). Data analysis was performed using FlowJo software (version 10.0).

ImageStream. Single brain cells were prepared as above. For preparation of peripheral blood mononuclear cells (PBMCs), peripheral blood $(600 \mu \mathrm{L})$ was collected into $100 \mu \mathrm{L} 1 \%$ heparin. PBMCs were separated with Ficoll paque-PLUS (17-1440-02, GE Healthcare) gradient centrifugation ( $800 \mathrm{~g}, 20$ minutes, $18^{\circ} \mathrm{C}$ ). The mononuclear cells in the interface were collected and washed with HBSS containing $1 \%$ FBS and 2 mM EDTA. Single brain cells or PBMCs were stained with an antibody cocktail (anti-pSTAT6-PE, 558252, BD Bioscience, 1:100; anti-Arg1-APC, IC5868A, R\&D Systems, 1:100; anti-CD45-PB, 48-045182, Thermo Fisher Scientific, 1:400; anti-CD11b-BV605, 563015, BD Bioscience, 1:400) or the appropriate isotype controls following the manufacturer's instruction (Thermo Fisher Scientific). For every $1 \times 10^{6}$ cells, $100 \mu \mathrm{L}$ antibody cocktail diluted in HBSS containing 1\% FBS and 2 mM EDTA (surface marker) or $1 \times$ permeabilization buffer (GAS002S100, Thermo Fisher Scientific) (intracellular marker) was used. 
Fluorochrome compensation was performed with single-stained OneComp eBeads (01-1111-41, Thermo Fisher Scientific). The gate for GFP was set up by using the cells collected from WT nonchimera mouse ischemic brain as a control. ImageStream analysis was performed using Amnis Imaging Flow Cytometer (MilliporeSigma). Data analysis was performed using IDEAS software (MilliporeSigma).

Behavioral tests. Sensorimotor functions were measured by rotarod and foot-fault tests $1 \mathrm{~d}-3 \mathrm{~d}$ before and 3d-14d after tMCAO. Cognitive function was analyzed with the Morris water maze test 21d-25d after tMCAO.

Rotarod test. Mice were pretrained for 3 consecutive days before tMCAO using an accelerated rotarod training paradigm, and data in the last training session were recorded as baseline. Tests were performed at $3 \mathrm{~d}, 5 \mathrm{~d}$, $7 \mathrm{~d}, 10 \mathrm{~d}$, and $14 \mathrm{~d}$ after tMCAO. Mice were placed on a rotating drum with a speed accelerating from 0-300 rpm within 5 minutes and maintained at this final speed. The trial ended if the animal fell off the rungs or gripped the device and spun around for 2 consecutive revolutions without attempting to walk on the rungs. The latency to fall or spin around on the rungs was recorded. Data were expressed as mean values from 3 trials.

Foot-fault test. Each mouse was placed on a stainless-steel grid floor $(20 \mathrm{~cm} \times 40 \mathrm{~cm}$ with a mesh size of $4 \mathrm{~cm}^{2}$ ) elevated $1 \mathrm{~m}$ above the floor. The total number of steps was counted for a videotaped 1-minute observation period. The number of forelimb foot-fault (when the forelimb fell through the grid) was recorded. Mice were pretrained for 3 consecutive days before tMCAO, and data in the last training session were recorded as baseline. Tests were performed at $3 \mathrm{~d}, 5 \mathrm{~d}, 7 \mathrm{~d}, 10 \mathrm{~d}$, and $14 \mathrm{~d}$ after $\mathrm{tMCAO}$.

Morris water maze test. Cognitive function was determined using a water maze test as described previously (12). Animals were tested at 21d-24d after tMCAO. Three trials were performed on each day. The time spent to reach the platform (learning phase) was recorded. Memory test was performed on day 25. Time spent in the goal quadrant where the platform was previously localized was recorded.

Reverse transcription-quantitative PCR (RT-qPCR). Total RNA was isolated from brains (ischemic hemisphere or sham brain) with the RNeasy Lipid Tissue Mini kit (74804, QIAGEN). Total RNA of primary cultured microglia/macrophages was isolated using the RNeasy Mini Kit (74106, QIAGEN) according to the manufacturer's instructions. RNA $(1 \mu \mathrm{g})$ was used to synthesize the first strand of cDNA using the Superscript First-Strand Synthesis System for RT-qPCR (11752, Invitrogen) according to the manufacturer's protocols. Program for reverse transcription was $25^{\circ} \mathrm{C}$ for 10 minutes, $50^{\circ} \mathrm{C}$ for 30 minutes, $85^{\circ} \mathrm{C}$ for 5 minutes, and $4^{\circ} \mathrm{C}$ for maintenance. PCR was performed on the Opticon 2 Real-Time PCR Detection System (Bio-Rad) using corresponding primers (Supplemental Table 1) and SYBR green PCR Master Mix (330503, QIAGEN). The program for real-time PCR was $95^{\circ} \mathrm{C} 15$ minutes, $\left(94^{\circ} \mathrm{C} 20\right.$ seconds $\left[20 \mathrm{~s}\right.$ ], $59^{\circ} \mathrm{C} 30 \mathrm{~s}, 72^{\circ} \mathrm{C}$ $30 \mathrm{~s}) \times 40$, melting curve from $50^{\circ} \mathrm{C}-92^{\circ} \mathrm{C}$, read every $0.2^{\circ} \mathrm{C}$, hold $2 \mathrm{~s}$, incubate at $8^{\circ} \mathrm{C}$. The cycle time values were normalized to GAPDH in the same sample as an internal control. The expression levels of the mRNAs were then reported as fold change vs. sham brain or vehicle-treated microglia/macrophages.

Primary microglia-enriched and macrophage-enriched cultures. Primary microglia-enriched culture were prepared from the whole brains of 1- to 3-day-old WT or STAT6-KO mouse pups as described previously (40).

Primary macrophage-enriched cultures were prepared from the BM of 8- to 10-week-old WT or STAT6KO mice. Monocytes were isolated using a mouse monocyte isolation kit (Stemcell Technologies) according to the manufacturer's instructions. Monocytes were seeded in $75 \mathrm{~cm}^{2}$ culture flasks $\left(2 \times 10^{7}\right.$ cells per flask) (Thermo Fisher Scientific) and cultured in the presence of macrophage CSF (M-CSF, $20 \mathrm{ng} / \mathrm{mL}$ ) to induce their differentiation into macrophage. Macrophages were collected at $7 \mathrm{~d}$ in vitro for further experiments. Culture medium for macrophage was RPMI1640 (Thermo Fisher Scientific) containing 10\% FBS (Sigma-Aldrich).

Primary cortical neuronal culture and induction of in vitro ischemia. Primary cortical neuronal cultures were prepared from C57BL/6J mouse E17 embryos as previously described (38). Neurons were seeded on polyD-lysine-coated (MilliporeSigma P6704) coverslips (Thermo Fisher Scientific) in 24-well plates (Thermo Fisher Scientific) $\left(3 \times 10^{5}\right.$ cells per well) with neural basal medium (Thermo Fisher Scientific) containing B27 (Invitrogen) and glutaMax (Thermo Fisher Scientific). In vitro ischemia was conducted at 10d in vitro as described (38). For neuron efferocytosis experiments, neurons were labeled with $1 \mu \mathrm{g} / \mathrm{mL}$ PI in FBS-free neural basal medium ( $1 \mathrm{~mL}$ PI staining medium to every $1 \times 10^{7}$ neurons) and incubated at $37^{\circ} \mathrm{C}$ for 10 minutes. Cells were then washed and added to microglia or macrophages.

Neuron-microglia/macrophage cocultures. Ten-day-old neurons cultured in 24 -well plate $\left(3 \times 10^{5} /\right.$ well $)$ were subjected to 90 minutes of OGD. Primary microglia/macrophage $\left(7.5 \times 10^{4} /\right.$ well $)$ were then seeded and cultured together with neurons for 24 hours after OGD in coculture medium $(250 \mu \mathrm{L}$ neural basal medium and $250 \mu \mathrm{L}$ DMEM/F12-based glia cell medium or RPMI1640-based macrophage culture medium). To inhibit phagocytic activity of microglia/macrophages, the cells were pretreated with $10 \mu \mathrm{M}$ Cytochalasin D (C8273, 
Sigma-Aldrich) for $1 \mathrm{~h}$ and then washed before coculturing. To analyze neuronal survival, neurons were fixed with 4\% paraformaldehyde and stained with anti-MAP2 antibodies (MAB3418, 1:300; MilliporeSigma). $\mathrm{MAP}^{+}$cells were defined as survival neurons. Images were processed with NIH ImageJ software by a blinded observer for the unbiased counting of automatically recognized cells.

Western blot. Protein isolation from cultured microglia or macrophages was performed as previously described (41). Western blots were performed using the standard SDS-polyacryamide gel electrophoresis method and enhanced chemiluminescence detection reagents (GE Healthcare). Antibodies against Arg1 (goat anti-Arg1, sc-18351, 1:500; Santa Cruz Biotechnology Inc.) and $\beta$-actin (mouse anti- $\beta$-actin, A2228, 1:20,000; Sigma-Aldrich) were used. Immunoreactivity was semiquantitatively analyzed by the ImageJ (NIH) software. Images of blots were cropped for presentation. Full-size images are presented in Supplemental Figure 12.

Efferocytosis assay. Primary cultured microglia or macrophages were plated on poly-D-Lysine-coated coverslips in 24-well plates $\left(2 \times 10^{5}\right.$ cells/well $)$ and cultured for 24 hours. $\mathrm{PI}^{+}$dead/dying neurons were added to microglia or macrophage at a ratio of 10:1 and incubated for 1-6 hours in DMEM (Thermo Fisher Scientific) containing 2\% FBS (Sigma-Aldrich). Cells were then rinsed with PBS and fixed in 4\% paraformaldehyde. F-actin of microglia or macrophage was then stained with Alexa Fluor488 phalloidin (A12379, 1:300 in PBS; Invitrogen) at room temperature in the dark for 1 hour. Images were captured with an Olympus confocal microscope. To analyze the function of Arg1 in efferocytosis, microglia or macrophages were treated with a specific Arg inhibitor N(G)-Hydroxy-L-arginine acetate salt (NOHA, $100 \mu \mathrm{M}$ for 1 hour). Inhibitor-treated cells were washed and subjected to the efferocytosis assay. Images were processed with NIH ImageJ software by a blinded observer for the unbiased counting of automatically recognized cells. In some experiments, phagocytic activity of WT and STAT6-KO microglia or macrophage was analyzed with fluorescence beads as described before (17). Cell survival before and after the efferocytosis assay was analyzed with lactate dehydrogenase (LDH) release (L7572, Pointe Scientific) in medium following the manufacturer's instructions.

Lentiviral infection of microglia/macrophages. To construct lentiviral vectors-overexpressing Arg1, GFP and the HA-tagged cDNA was inserted into the lentiviral transfer vector pFPWS C-terminal. The constructed transfer vectors were transformed into Stbl3 E. coli and then isolated using the EndoFree Plasmid Maxi Kit (QIAGEN). Large-scale production of the virus was performed using a protocol described previously (42). In brief, a plasmid mixture containing pCMV $\delta$ R8.9 (packaging construct), pVSVG (envelope plasmid), and pFPWS-Arg1 (transfer vector) was suspended in $\mathrm{CaCl}_{2}$ $(250 \mathrm{mM})$ and the same volume of $2 \times$ BES buffer $(\mathrm{pH} 6.95)$ was added. The DNA-CaCl ${ }_{2}$ precipitate was added to human kidney 293 FT cells and allowed to incubate for 12 hours before switching to fresh culture medium. The supernatant was collected $48 \mathrm{~h}$ hours after transfection, filtered through a $0.45-\mu \mathrm{m}$ filter flask, and centrifuged at $54,000 \mathrm{~g}$ for 2 hours. The pellet was suspended in $3 \mathrm{~mL}$ of DMEM, and viruses were further purified by sucrose gradient ultracentrifugation, loaded on the top of $2 \mathrm{~mL}$ of $20 \%$ sucrose solution, and centrifuged at $59,000 \mathrm{~g}$ for 2 hours. The resulting pellet was resuspended in $200 \mu \mathrm{L}$ of OPTI-MEM, aliquoted, and stored at $-80^{\circ} \mathrm{C}$. The titer of the vector stock was determined by infection of 293 cells to calculate the transduction unit.

The microglia or macrophage cultures were infected for 3d with Lenti-Arg1 or the control vectors (Lenti-GFP). The overexpression of Arg1 was confirmed by Western blot analysis of Arg1 and immunostaining of HA tag.

Statistics. Results were presented as mean \pm SD. Sample sizes for animal studies were determined by power calculations based on pilot studies or the literature. GraphPad Prism software (version 6.0) was used for statistical analyses, unless otherwise indicated. The 2-tailed Student's $t$ test was used for comparison of 2 groups. The differences in means among multiple groups were analyzed using 1-way ANOVA for continuous variables with normal distribution. Differences in means across groups with repeated measurements over time were analyzed using the repeated-measures 2-way ANOVA. When 1- or 2-way ANOVA showed significant differences, pair-wise comparisons between means were tested by post hoc Bonferroni tests. When parametric assumptions were not met, the nonparametric 2-tailed Mann-Whitney $U$ test was applied. In all analysis, $P \leq 0.05$ was considered statistically significant.

Study approval. The acquisition and handling of human tissue were approved by the CORID (no. 914). All animal procedures were approved by the University of Pittsburgh IACUC and performed in accordance with the Guide for the Care and Use of Laboratory Animals (National Academies Press, 2011), Stroke Treatment Academic Industry Roundtable criteria, and Stroke Therapy Academic Industry Roundtable group guidelines for preclinical evaluation of stroke therapeutics. 


\section{Author contributions}

WC, XD, Jie Chen, JZ, MX, LZ, BY, WZ, MR, TN, JK, and YS performed the experiments and collected and analyzed data. WC, XH, and Jun Chen designed the experiments. WC and XH wrote the manuscript. YS, RAS, XH, and Jun Chen critically revised the manuscript. The order among co-first authors is determined by their contribution to performing experiments and writing the manuscript.

\section{Acknowledgments}

This work was supported by grants from the NIH (NS092618 to XH; AG005133 to JK) and VA (I01 BX003651 to XH, I01 BX003377 to Jun Chen). Jun Chen is a recipient of the VA Senior Research Career Scientist Award. This work benefitted from ImageStreamX MARKII grant NIH 1S10OD019942. We thank Amanda Smith for editorial assistance.

Address correspondence to: Jun Chen or Xiaoming Hu, Department of Neurology, University of Pittsburgh, 200 Lothrop Street, SBST 507, Pittsburgh, Pennsylvania 15213, USA. Phone: 412.648.1263; Email: chenj2@upmc.edu (JC). Phone: 412.648.8991; Email: hux2@upmc.edu_(XH).

1. An C, et al. Molecular dialogs between the ischemic brain and the peripheral immune system: dualistic roles in injury and repair. Prog Neurobiol. 2014;115:6-24.

2. Shichita T, Ito M, Yoshimura A. Post-ischemic inflammation regulates neural damage and protection. Front Cell Neurosci. 2014;8:319.

3. Liesz A, et al. DAMP signaling is a key pathway inducing immune modulation after brain injury. J Neurosci. 2015;35(2):583-598.

4. Arandjelovic S, Ravichandran KS. Phagocytosis of apoptotic cells in homeostasis. Nat Immunol. 2015;16(9):907-917.

5. Patel AR, Ritzel R, McCullough LD, Liu F. Microglia and ischemic stroke: a double-edged sword. Int J Physiol Pathophysiol Pharmacol. 2013;5(2):73-90.

6. García-Culebras A, et al. Myeloid cells as therapeutic targets in neuroinflammation after stroke: Specific roles of neutrophils and neutrophil-platelet interactions. J Cereb Blood Flow Metab. 2018;38(12):2150-2164.

7. Hu X, et al. Microglial and macrophage polarization — new prospects for brain repair. Nat Rev Neurol. 2015;11(1):56-64.

8. Kurisu K, et al. Triggering receptor expressed on myeloid cells-2 expression in the brain is required for maximal phagocytic activity and improved neurological outcomes following experimental stroke. J Cereb Blood Flow Metab. 2018;null(10):27167 8X18817282.

9. Elliott MR, Koster KM, Murphy PS. Efferocytosis Signaling in the Regulation of Macrophage Inflammatory Responses. J Immunol. 2017;198(4):1387-1394.

10. Brunn A, et al. IL-10, IL-4, and STAT6 promote an M2 milieu required for termination of P0(106-125)-induced murine experimental autoimmune neuritis. Am J Pathol. 2014;184(10):2627-2640.

11. Qin H, Holdbrooks AT, Liu Y, Reynolds SL, Yanagisawa LL, Benveniste EN. SOCS3 deficiency promotes M1 macrophage polarization and inflammation. J Immunol. 2012;189(7):3439-3448.

12. Liu X, et al. Interleukin-4 Is Essential for Microglia/Macrophage M2 Polarization and Long-Term Recovery After Cerebral Ischemia. Stroke. 2016;47(2):498-504.

13. Chen HC, Reich NC. Live cell imaging reveals continuous STAT6 nuclear trafficking. J Immunol. 2010;185(1):64-70.

14. Jung JE, et al. STAT-dependent upregulation of 12/15-lipoxygenase contributes to neuronal injury after stroke. J Cereb Blood Flow Metab. 2015;35(12):2043-2051.

15. Bushnell CD, et al. Sex differences in stroke: Challenges and opportunities. J Cereb Blood Flow Metab. 2018;38(12):2179-2191.

16. Garcia-Bonilla L, et al. Endogenous Protection from Ischemic Brain Injury by Preconditioned Monocytes. J Neurosci. 2018;38(30):6722-6736.

17. Hu X, et al. Microglia/macrophage polarization dynamics reveal novel mechanism of injury expansion after focal cerebral ischemia. Stroke. 2012;43(11):3063-3070.

18. Fenn AM, Hall JC, Gensel JC, Popovich PG, Godbout JP. IL-4 signaling drives a unique arginase+/IL-1 $\beta+$ microglia phenotype and recruits macrophages to the inflammatory CNS: consequences of age-related deficits in IL-4R $\alpha$ after traumatic spinal cord injury. J Neurosci. 2014;34(26):8904-8917.

19. Erdö F, Trapp T, Mies G, Hossmann KA. Immunohistochemical analysis of protein expression after middle cerebral artery occlusion in mice. Acta Neuropathol. 2004;107(2):127-136.

20. Zhu J, Guo L, Watson CJ, Hu-Li J, Paul WE. Stat6 is necessary and sufficient for IL-4's role in Th2 differentiation and cell expansion. J Immunol. 2001;166(12):7276-7281.

21. Moreno JL, Mikhailenko I, Tondravi MM, Keegan AD. IL-4 promotes the formation of multinucleated giant cells from macrophage precursors by a STAT6-dependent, homotypic mechanism: contribution of E-cadherin. J Leukoc Biol. 2007;82(6):1542-1553.

22. Brown GC, Neher JJ. Microglial phagocytosis of live neurons. Nat Rev Neurosci. 2014;15(4):209-216.

23. Szanto A, et al. STAT6 transcription factor is a facilitator of the nuclear receptor PPAR $\gamma$-regulated gene expression in macrophages and dendritic cells. Immunity. 2010;33(5):699-712.

24. Berry A, et al. IL-13 induces expression of CD36 in human monocytes through PPARgamma activation. Eur J Immunol. 2007;37(6):1642-1652.

25. Serghides L, Kain KC. Peroxisome proliferator-activated receptor gamma-retinoid X receptor agonists increase CD36-dependent phagocytosis of Plasmodium falciparum-parasitized erythrocytes and decrease malaria-induced TNF-alpha secretion by 
monocytes/macrophages. J Immunol. 2001;166(11):6742-6748.

26. Zhao XR, Gonzales N, Aronowski J. Pleiotropic role of PPAR $\gamma$ in intracerebral hemorrhage: an intricate system involving Nrf2, RXR, and NF-кB. CNS Neurosci Ther. 2015;21(4):357-366.

27. Galès A, et al. PPAR $\gamma$ controls Dectin-1 expression required for host antifungal defense against Candida albicans. PLoS Pathog. 2010;6(1):e1000714.

28. Murphy PS, et al. CD73 regulates anti-inflammatory signaling between apoptotic cells and endotoxin-conditioned tissue macrophages. Cell Death Differ. 2017;24(3):559-570.

29. A-Gonzalez N, et al. Apoptotic cells promote their own clearance and immune tolerance through activation of the nuclear receptor LXR. Immunity. 2009;31(2):245-258.

30. Osorio EY, Travi BL, da Cruz AM, Saldarriaga OA, Medina AA, Melby PC. Growth factor and Th2 cytokine signaling pathways converge at STAT6 to promote arginase expression in progressive experimental visceral leishmaniasis. PLoS Pathog. 2014;10(6):e1004165.

31. Yang Q, et al. Cross talk between histone deacetylase 4 and STAT6 in the transcriptional regulation of arginase 1 during mouse dendritic cell differentiation. Mol Cell Biol. 2015;35(1):63-75.

32. Cherry JD, Olschowka JA, O'Banion MK. Neuroinflammation and M2 microglia: the good, the bad, and the inflamed. J Neuroinflammation. 2014;11:98.

33. Quirié A, et al. Effect of stroke on arginase expression and localization in the rat brain. Eur J Neurosci. 2013;37(7):1193-1202.

34. Truettner JS, Bramlett HM, Dietrich WD. Posttraumatic therapeutic hypothermia alters microglial and macrophage polarization toward a beneficial phenotype. J Cereb Blood Flow Metab. 2017;37(8):2952-2962.

35. Greenhalgh AD, Passos Dos Santos R, Zarruk JG, Salmon CK, Kroner A, David S. Arginase-1 is expressed exclusively by infiltrating myeloid cells in CNS injury and disease. Brain Behav Immun. 2016;56:61-67.

36. Cherry JD, Olschowka JA, O'Banion MK. Arginase $1+$ microglia reduce A $\beta$ plaque deposition during IL- $1 \beta$-dependent neuroinflammation. J Neuroinflammation. 2015;12:203.

37. Li P, et al. Adoptive regulatory T-cell therapy protects against cerebral ischemia. Ann Neurol. 2013;74(3):458-471.

38. Yang Y, et al. ST2/IL-33-Dependent Microglial Response Limits Acute Ischemic Brain Injury. J Neurosci. 2017;37(18):4692-4704

39. Zhang Q, et al. The interleukin-4/PPAR $\gamma$ signaling axis promotes oligodendrocyte differentiation and remyelination after brain injury. PLoS Biol. 2019;17(6):e3000330.

40. $\mathrm{Hu} \mathrm{X}$, et al. Macrophage antigen complex-1 mediates reactive microgliosis and progressive dopaminergic neurodegeneration in the MPTP model of Parkinson's disease. J Immunol. 2008;181(10):7194-7204.

41. Shi Y, et al. Rapid endothelial cytoskeletal reorganization enables early blood-brain barrier disruption and long-term ischaemic reperfusion brain injury. Nat Commun. 2016;7:10523.

42. Stetler RA, et al. Hsp27 protects against ischemic brain injury via attenuation of a novel stress-response cascade upstream of mitochondrial cell death signaling. J Neurosci. 2008;28(49):13038-13055. 